\title{
Semi-global output feedback nonlinear stabilization of variable speed grid connected direct drive wind turbine generator systems
}

\author{
Ali Abdul Razzaq Al Tahir ${ }^{1}$
}

Received: 3 May 2016 / Revised: 15 November 2016 / Accepted: 17 November 2016 / Published online: 27 December 2016

(C) The Author(s) 2016. This article is published with open access at Springerlink.com

\begin{abstract}
The goal of the present work is to achieve nonlinear semi-global output feedback stabilization of variable speed grid connected direct drive wind turbine generator DDWTG systems under wind energy conversion systems to provide the electricity demand for electrical networks. The study will address the sensorless control of variable speed wind energy generation systems, namely optimized rotor speed and direct-axis current control to determine the most appropriate structure and to improve both robustness and reliability of this kind of distributed generators. The proposed nonlinear observer is robust with respect to measurements error and perturbations of the sampling schedule. The simulation results of the case study support the presented main theorem that reflects the effect of measurement errors and the improvement with respect to global convergence properties. The novelty of the present study resides in taking into account the difficulties and control problem complexity that faced the nonlinear output feedback control undergo:
\end{abstract}

Preliminaries and Notations

Throughout this paper, |.| means the absolute value. Let us consider $R \stackrel{\text { def }}{=}(-\infty, \infty), R^{+} \stackrel{\text { def }}{=}(0, \infty)$ positive real number space, $R_{0}^{+}=$ $[0, \infty]$, and let us define $\|$.$\| be the Euclidean norm or the induced$ matrix norm. For $\mathrm{p}, \mathrm{q}, \mathrm{n}, \mathrm{m} \in N$, set of natural numbers, $R^{p \times q}$ represents the set of real matrices of order $\mathrm{p} \times \mathrm{q}$ and $I_{p} \in I R^{p \times p}$ stands for the identity matrix of order $\mathrm{p} \times \mathrm{p}$. The notation $\|P\|$, for $P \in R^{p \times q}$, represents the $L_{2}$-norm of $\mathrm{P}$ and $X^{T}$, represent the transposed vector of $X$. One say that $\alpha I_{n} \leq P \leq \beta I_{n}$ where $\mathrm{P} \in R^{n \times n}$ if, $\lambda \min (P) \geq \alpha$ and $\lambda \max (P) \geq \beta$ where $\lambda \min ($.$) and$ $\lambda \max$ (.) denote, respectively the smallest and the biggest eigenvalues of the square matrix. In all this study, the initial boundary time is called $t_{0} \in[0, \infty)$.

Ali Abdul Razzaq Al Tahir

razzaqali2007@yahoo.com; ali.altahir@uokerbala.edu.iq

1 Electrical Engineering Department, College of Engineering, University of Kerbala, Ministery of Higher Education and Scientific Research, Kerbala, Iraq (i) the proposed system nonlinearity and loss the observability of sensorless PMSG near the singularity point of the rotor speed; (ii) inaccessibility measurements for all system state variables; (iii) maximum power point tracking of optimum reference angular rotor speed; (IV) magnetizing and demagnetizing direct-axis current at standstill; (V) the proposed semi-global observer has dynamic high-gain design parameter DHGO. The simulation results of variable speed grid connected DDWTG, in general, confirm the main theorem developed under DHGO parameter and lead us to enlarge and prolongation the admissible sampling period using nonlinear Lyapunov stability control techniques.

Keywords $A C / D C / A C$ double converters $\cdot M P P T \cdot$ Semiglobal observer $\cdot$ Sampled measurements $\cdot$ Backstepping control $\cdot$ Lyapunov stability tools

\section{List of symbols}

$R_{a} \quad$ Resistance of armature windings for PMSG

$(\Omega)$

$L_{a} \quad$ Armature winding inductance for PMSG (H)

$V_{g \alpha}, V_{g \beta} \quad \alpha-\beta$ axis of wind turbine generator voltage (V)

$I_{g \alpha}, I_{g \beta} \quad \alpha-\beta$ axis of wind turbine generator current (A)

$\omega_{g} \quad$ Angular velocity of the generator rotor $(\mathrm{rad} / \mathrm{s})$

$T_{m} \quad$ External input mechanical torque ( $\mathrm{Nm}$ )

$\mathrm{p} \quad$ Number of pole pairs

$J \quad$ Total inertia of the WTG rotor $\left(\mathrm{Nm} / \mathrm{rad} / \mathrm{s}^{\mathrm{s}}\right)$

$F \quad$ Total viscous friction of WTG rotor $(\mathrm{N} \mathrm{m} / \mathrm{rad} / \mathrm{s})$

$\theta_{m} \quad$ Mechanical rotor position angle (rad)

$\psi_{P M} \quad$ Permanent Magnent flux constant (Wb)

$C_{\text {bus }} \quad$ DC link capacitor (F)

$P_{m} \quad$ Mechanical output power (W)

$\rho \quad$ Air density (1.225) $\left(\mathrm{kg} / \mathrm{m}^{3}\right)$ 


$\begin{array}{ll}C_{p} & \text { The power coefficient (unit less) } \\ C_{f i l} & \text { Capacitance of LCL filter (F) } \\ A & \text { The swept area of the turbine blade }\left(\mathrm{m}^{2}\right) \\ V_{w} & \text { The wind speed velocity }(\mathrm{m} / \mathrm{s}) \\ \mathrm{D} & \text { The rotor diameter }(\mathrm{m}) \\ L_{f i l} & \text { Inductance of LCL filter }(\mathrm{H})\end{array}$

\section{Introduction}

Recently, wind energy is a promised energy for the coming years and pollution-free energy, which can help us to reduce the carbon emissions. Also, it will reduce the electricity bill for household operations. Wind energy reduces national reliance on carbon-based fuels, and it will decrease the production of greenhouse gas emissions that will reflect positive effects on environments, limits the pollution and tackle climate change, in the world. DDWTG can be used to generate electricity in remote location such as mountains and remote countryside [1].

Although, several works deal with wind turbine simulator have been done in the past few years. They have been some studies on wind turbine simulator based on DC generators, inductions generators and permanent magnet synchronous generators. Moreover, to avoid the need of tach generators and differentiation of rotor position and to guarantee redundancy of information, few solutions have been suggested to observe simultaneously the states trajectories, perturbation and all mechanical and electrical parameters [2].

A new variable speed wind energy control system with a permanent magnet synchronous generator and impedance source inverter has been proposed in [3]. Characteristics of impedance source inverter are used for maximum power point tracking control and delivering power to the grid simultaneously. Two control methods are proposed for delivering power to the grid: capacitor voltage control and DC- bus voltage control. The maximum power point tracking MPPT algorithm used in this method is based on relation between the DC voltage and the generator speed. In the WECS, wind turbine can operate with either variable speed or fixed speed. For fixed speed wind generation system, because of the generator is directly connected to the grid, the turbulence of the wind will result in power variations, and so affect the power quality in the grid, whereas for variable speed generation system, the generator is controlled by power electronic converter. So, variable speed wind energy conversion systems have many advantages over fixed speed generation, such as maximum power point tracking control method, increased power capture, power quality, improved efficiency and they can be controlled in order to reduce aerodynamic noise and mechanical stress.
With the development of power electronics technology, it's possible to control the rotor speed, to increase wind energy production and to reduce drive train loads. Thus the variable speed wind turbine generator system is becoming the most important and fastest growing application of wind generation system. The use of the PMSG is becoming more and more common for several reasons such as: very high torque can be achieved at low speeds because PMSG is connected directly to the turbine without gearbox; lower operational noise is achieved; no significant losses are generated in the rotor and external excitation current is not needed. So, the efficiency of a PMSG based WECS has been assessed higher than other generators and PMSG is an attractive choice for variable-speed generation system. In the case of PMSG based WECS, because of the advance of power electronic technology, decreasing equipment costs [4]. Wind power production has been under the main focus for the past decade in power production and tremendous amount of research work is going on renewable energy, specifically on wind power extraction. Wind power provides an eco-friendly power generation and helps to meet the national energy demand when there is a diminishing trend in terms of non-renewable resources. As already mentioned, mechanical sensors based solutions are most costly and unreliable. Then, state observers turn out to be a quite natural alternative to get estimates of mechanical variables using only measurable electrical state variables [5].

There are many literatures concerning with the design of continuous-time high gain observers for example as in [6], the authors proposed a global observer with the global Lipschitz condition of the system. Nevertheless, this assumption is removed through the Lipschitz extension technique when the semi-global observer is proposed. For continuous-time systems introduced by [7-9] are designed in order to address the issue of the sensitivity to measurements error inherent to these kinds of observers. A robust high-gain observer for nonlinear systems to provide a solution to the noise sensitivity of high-gain observers, which behaves well with respect to noise and the high gain Extended Kalman Filter EKF that is performant with respect to large perturbations, was proposed by [10].

Several studies had been addressed the problem of controlling power generation in variable speed wind turbines, such as sliding mode control and backstepping control strategies have been proposed to ensure stable operation in different operating regions [11-13]. Many nonlinear control techniques have been proposed, such as fuzzy logic-control, backstepping control technique, differential Flatness based control, and sliding mode control. However, most of the above works need continuous-time measurements of the AC voltages, the $\mathrm{AC}$-currents, and the dc-voltage. This requires many of voltage and current sensors, which will increase system complexity, cost, space and reduces system reliability of operation. 
A simple control strategy for the operation of a variable speed stand-alone wind turbine with a permanent magnet synchronous generator (PMSG) to meet maximum power extraction from the available wind power through DCDC bidirectional buck-boost converter, which is connected between batteries bank and DC-link voltage, is used to maintain the DC-bus voltage at a constant value claimed by [14]. Existing MPPT methods can be separated in two categories. The first one includes methods based on the explicit use of the wind turbine power characteristics, which necessitate online measurements of wind velocity and (turbine/PMSG) rotor velocity [15]. In fact, the required wind velocity measurement is a kind of average value of wind velocity along the turbine blade which is not easy to measure. This drawback is overcome in [16] where the proposed MPPT method involves a Kalman predictor estimating the load/turbine torque based on rotor speed measurements. The whole control design, including the Kalman predictor, is based on a linear approximation of the wind turbine systems and no formal analysis is made therefor the proposed control strategy was not proved. The second category of MPPT methods, using perturbation observation technique, without needing turbine characteristics as provided in $[17,18]$. These methods are most suitable for small scale wind turbine generator systems.

Output feedback control using high-gain observers as well as measurement noise for a class of nonlinear systems was provided by [19], A brief introduction to high-gain observers in nonlinear feedback control introduced by [20], with emphasis on the peaking phenomenon and the role of control saturation in dealing with it. The paper surveys recent results on the nonlinear separation principle, conditional servo compensators, extended high-gain observers, performance in the presence of measurement noise and sampled-data control. A semi-global stabilization through output-feedback for a class of uncertain nonlinear systems was presented by [21]. An output-feedback controller is constructed for the systems with the appropriate choice of design parameters; this controller can make the closed-loop system globally attractive and semi-globally exponentially stable at the region of attraction.

In this study, semi-global sampled high-gain observer design having dynamic HGO parameter is proposed to get online estimates of the generator rotor speed, rotor position angle and external input generator torque from the measurements of stator voltages and output currents. The rotor position is derived from the estimated rotor fluxes in $(\alpha-\beta)$ stationary reference frame without needing initial rotor position detection. The major contributions of this work can be summarized as follows:

1. The successful construction of mathematical model of a variable speed grid connected direct drive wind tur- bine generator system and integrated it to the electrical networks.

2. Maximum power point extraction for best generator rotor angular speed.

3. On line estimation of the mechanical states variables, which are rotor angular speed, aerodynamic generator torque, taking into accounts the electrical variables (output currents and input control voltages) are accessible for measurements.

4. Estimation of rotor position angle whenever the estimates of the rotor flux vector $\left[\hat{\psi}_{\mathrm{r} \alpha}, \hat{\psi}_{\mathrm{r} \beta}\right]^{T}$ in stationary reference frame become available.

5. Best selection of direct-axis stator current component to ensure cancelation of reluctance torque and provide the maximum torque i.e., regulating the current $i_{d}$ to track a reference value $i_{\text {dref }}$. With this control, the generator torque is coordinated with the $q$-axis current,

6. Regulation of DC bus voltage, control of active and reactive power, and satisfying unity power factor correction under variable speed wind condition.

One of the challenges faced this work that the electromagnetic torque is not direct output injected and another difficulty that the stator and rotor current vector is inaccessible to measurements all the time. Only sampled-data measurements are presently available at each sampling instant. This paper will focus on the generating system shown in Fig. 2, which consists of a PMSG that converts wind power into output voltage whose amplitude and frequency vary accordingly with wind velocity. The three-phase variable frequency, voltage generated from the wind turbine is rectified using a rectifier-inverter (AC/DC/AC) IGBT PWM converter), connected through a DC bus power transfer. The $\mathrm{AC}$ side of the rectifier is connected to the PMSG stator, the inverter DC/AC output is tied to the electrical grid via an inductive filter that performs the filtering of current harmonics due to the inverter switching actions. The PMSG is controlled through the above power converter that switches the phases depending on the generator rotor position. An observer based on sampled-data mechanism is used to reconstruct the rotor speed actuator and the control laws are reconfigured using the estimated state information.

This paper is organized as follows. Section 2 introduces the mathematical model for variable speed DDWT system, Sect. 3 presents state feedback nonlinear controller design objectives. Section 4 deals with DC voltage regulator and reactive power control. Section 5 focuses on semi-global output feedback nonlinear controller design; meanwhile, Sect. 6 stability convergence analysis of the proposed semi-global sampled HGO observer are discussed. Simulation results and verifications are shown in Sect. 7. Finally, the conclusion and remarks are drawn in Sect. 8. 


\section{Mathematical formulation for variable speed DDWTG}

\subsection{Modeling of wind turbine}

Some of wind turbines do not contain a gearbox and instead use a direct drive mechanism to produce power from the generator, which converts the aerodynamic energy to mechanical, electric energy and delivers it to the distribution system. As the wind velocity varies, the power captured which converted and transmitted to electricals load also varies [22]. The transmitted power is generally deduced from the wind power, using the power coefficient $C_{p}$ as:

$$
\begin{aligned}
P_{m} & =0.5 \rho A C_{p} V_{w}^{3} \\
C_{p} & =c_{1} \times\left(c_{2} \frac{1}{\lambda_{0}}-c_{3} \theta_{p}-c_{4}\right) e^{-c_{5} \frac{1}{\lambda_{0}}+c_{6} \lambda} \\
\lambda & =\frac{R \omega_{r}}{V_{w}}, \frac{1}{\lambda_{0}}=\frac{1}{\lambda+0.08 \theta_{p}}-\frac{0.035}{1+\left(\theta_{p}\right)^{3}} \\
V_{w} & =V_{b}+V_{g}+V_{r}+V_{\text {noise }}
\end{aligned}
$$

where, $P_{m}$ is the mechanical output power in watt, the air density $\rho$ in $\mathrm{kg} / \mathrm{m}^{3} . C_{p}$ (called the power coefficient or wind turbine efficiency), is a nonlinear function of tip speed ratio $\lambda=R \omega_{r} / V_{w}$, unit less, $A=\pi D^{2} / 4$ is the swept and recovered area of the wind turbine blades in $\mathrm{m}^{2}, \mathrm{D}$ is rotor diameter in $\mathrm{m}$, and $V_{w}$ is the wind velocity $(\mathrm{m} / \mathrm{s})$. With aerodynamic wind turbine coefficients, $\mathrm{c}_{1}, \mathrm{c}_{2}, \mathrm{c}_{3}, \mathrm{c}_{4}, \mathrm{c}_{5}$ and $c_{6}$. Wind model chosen for this study is consists of four components of wind speed $(\mathrm{m} / \mathrm{s})$, which are, $V_{b}, V_{g}, V_{r}$ and $V_{\text {noise. }}$. The base component is a constant speed and wind gust component can be usually represented as a sinusoidal function. The noise component may occur from wind acceleration and turbulence.

Wind turbine comprises of the blades and nacelle. The nacelle houses, electrical control systems, mechanical brake, electromagnetic brake and the PMSG. The blades drive the generator round which generates the electricity. Inverter as the voltage from the generator is different to that of the grid; the power from the generator needs to be converted to DC. The inverter then changes the output ensuring it is suit able for the local grid system [23]. The ideal power curve exhibits three different regions with distinctive generation objectives. At low wind velocity region, the available power is lower than rated power. The available power is defined as the power in the wind passing through the rotor area multiplied by the best power coefficient; $C_{p-o p t}(\lambda, \beta)<1$. So, the generation objective in first region is to extract all the available power. Therefore, the ideal power curve in this region follows a cubic parabola defined by system (1). On the other hand, the generation goal in the high wind velocity is to limit the generated power below its rated value to avoid over loading.

In this region, the available power exceeds rated power; therefore the wind turbine must be operated with efficiency lower than $C_{p-o p t}$. Finally, second region is actually a transition between the optimum power curve of first region and the constant power line of third region. In second region, rotor speed is limited to maintain acoustic noise emission within admissible levels and to keep centrifugal forces below values tolerated by the rotor.

\subsection{Sensorless speed reference optimization approach}

This study is looking for maximizing DDWTG efficiency at low wind velocity; the PMSG will be connected to the blades through a MPPT controller and optimization system instead of step-up gear box that will help us to overcome the cyclic maintenance for gear box. Specifically, the optimizer is expected to compute online the best speed value $\omega_{\text {opt }}$ so that, if the turbine rotor speed $\omega$ is made equal to $\omega_{\text {opt }}$ then, maximal wind energy is captured, and transmitted to the circuit utility throughout wind turbine generator WTG [24]. The speed-reference optimizer design is based on the turbine power characteristic and features the fact that it does not require wind velocity measurement. Figure 1, demonstrates turbine mechanical power versus rotor speed for variable wind velocities. The aerodynamic behavior of a wind turbine is described by the power coefficient curve $C_{p}$, so that it must be defined for each wind turbine. Structure of wind energy conversion system using PMSG is described in Fig. 2, which operates according to the well-known PWM fundamental. A coupling LCL filter is used to connect the grid side converter to the electrical grid. The aerodynamic behavior of a wind turbine is described by the power coefficient curve $C_{p}$, so that it must be defined for each wind turbine. Structure of wind energy conversion system using PMSG is described in Fig. 1, which operates according to the well-known PWM fundamental. Thus, when the wind velocity changes, the speed of PMSG is controlled to follow the maximum power point trajectory and, the optimum rotational speed of the generator can be simply estimated as follows:

$\omega_{o p t}=\frac{V_{w} \lambda_{o p t}}{D / 2}$

The optimum extracted power curve from WTG is defined as:

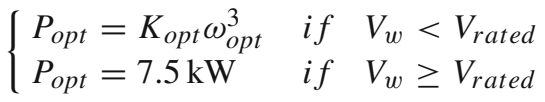

Consequently, the MPPT sensorless control evaluates the best wind velocity of PMSG and then regulating the wind 


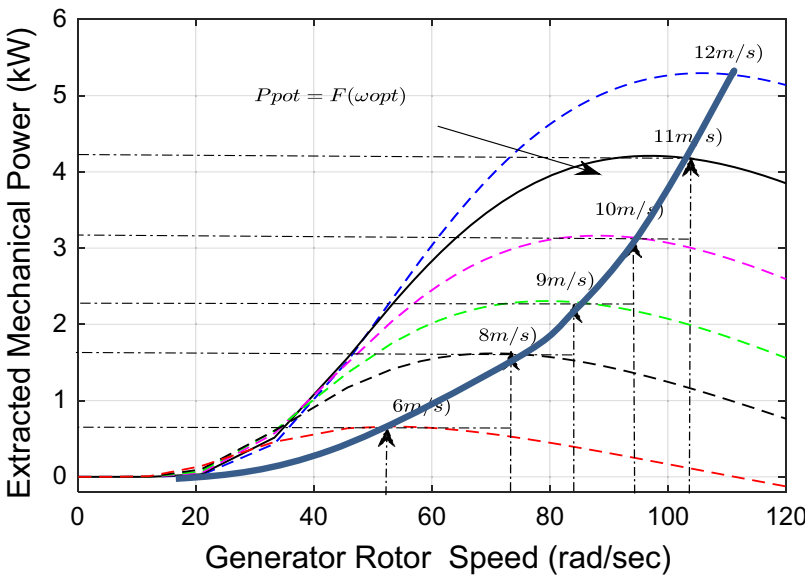

Fig. 1 Power speed characteristics for different

turbine generator speed in different wind velocities such that the maximum power is taken out. If the wind velocity reached the rated value of wind turbine, the system of pitch angle controller enters in operation to overcome turbine damage from excessive wind speed. Therefore, by reducing the power coefficient, both the generated power and generator rotor speed are maintained for above rated wind velocities. So, the blade pitch angle, will increase until the wind turbine stays at the rated speed. It is seen that for any wind velocity value, say $V_{w}$, there is a unique couple $\left(\omega_{i}, P_{i}\right)$ that involves the largest extractable power. A number of such couples have been collected from Fig. 1 and interpolated to get seventh degree polynomial function $\omega_{\text {opt }} \triangleq F\left(P_{\text {opt }}\right)$. Let the obtained polynomial given by [25]:
$F\left(P_{o p t}\right) \triangleq \omega_{o p t}=\sum_{i=0}^{8} d_{i} P^{i}$

The function $F\left(P_{o p t}\right)$ defines the speed-reference optimizer.

One can see on Fig. 1, which transmitted wind power $P_{0}$ corresponds to this couple $\left(V_{w}^{0}, \omega_{0}\right)$. The polynomial coefficients have values are listed in Table 1 . As there are no cheap and unreliable sensors of these variables, the above controller will remain useless. So, an observer is developed providing accurate estimates of the non-measurement variables. The main purpose of the present section is to design sampled high gain observer and use it to build up an output feedback controller that does not need measuring the un-sensed state variables.

\subsection{Modeling of the PMSG-AC/DC converter}

The controlled system is demonstrated in Fig. 2. It consists of a combination, PMSG-rectifier, on one hand, and a singlephase DC/AC inverter on the other hand. The rectifier is an $\mathrm{AC} / \mathrm{DC}$ converter, like the DC/AC rectifier, according to the known Pulse Width Modulation (PWM) principle. A capacitor connected on the DC side acts as the DC voltage source. To simplify the mathematical representation of PMSG, one shall use Clarke's transformation from $a b c / d-q$ coordinates, since $(d-q)$ representation is useful for nonlinear controller design because of its simplicity. In this study, one considers the cases of non-saliency rotor $\left(L_{d}=L_{q}=L_{a}\right)$. The dynamics of PMSG in $(d-q)$ reference frame is given by a set of differential equations as follows [26]:

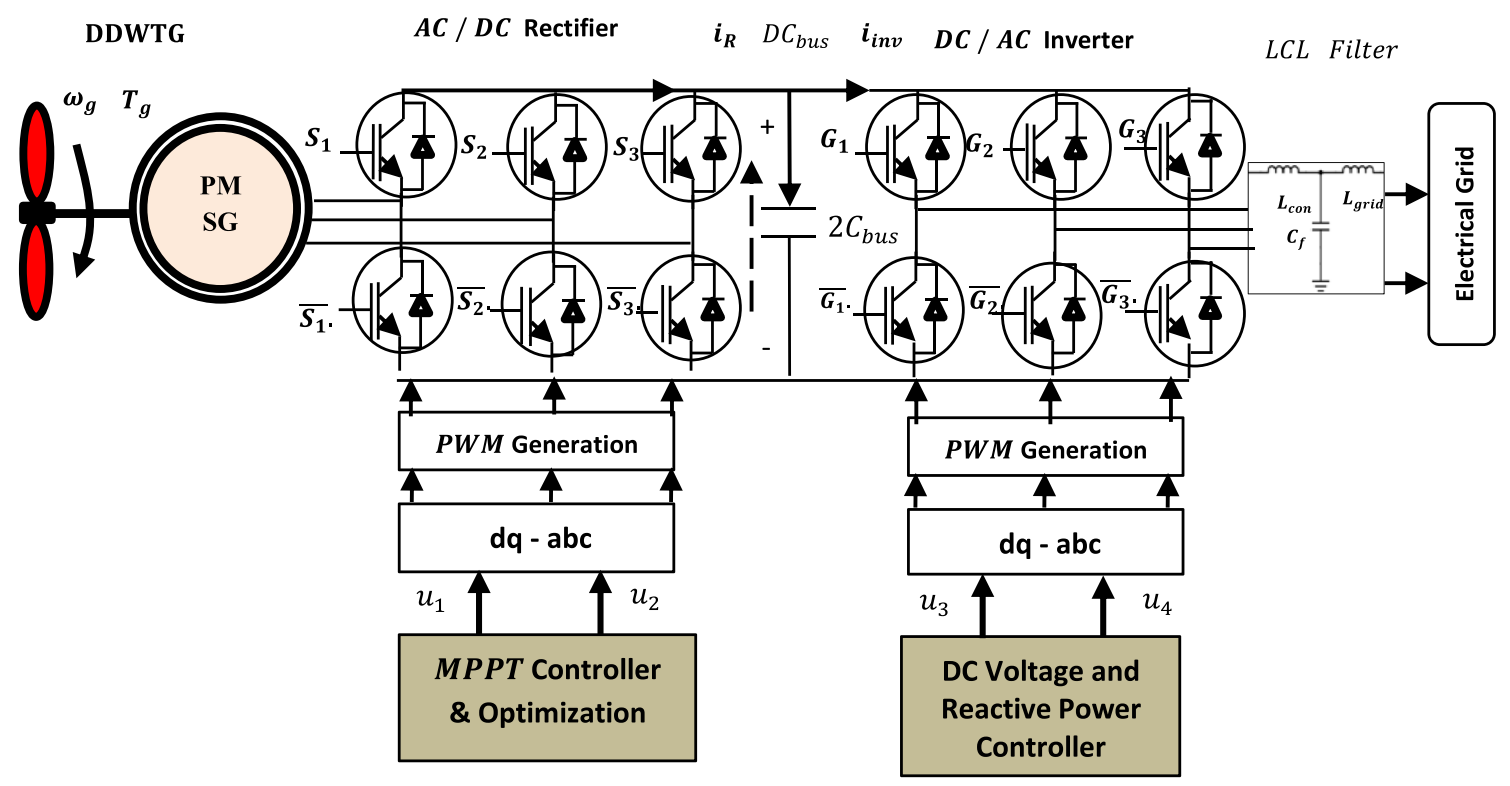

Fig. 2 General topology of wind control system 
Table 1 Polynomial interpolation coefficients

\begin{tabular}{lllll}
\hline$d_{0}$ & $d_{1}$ & $d_{2}$ & $d_{3}$ & $d_{4}$ \\
\hline 15.807 & -0.120 & 0.019 & -0.00031 & $-2.234 \mathrm{e}-05$ \\
\hline$d_{5}$ & $d_{6}$ & $d_{7}$ & $d_{8}$ & $d_{5}$ \\
\hline $9.441 \mathrm{e}-07$ & $-1.425 \mathrm{e}-08$ & $9.529 \mathrm{e}-11$ & $-2.348 \mathrm{e}-13$ & $9.441 \mathrm{e}-07$ \\
\hline
\end{tabular}

$u_{g d} \triangleq u_{2} V_{d c}, \quad u_{g q} \triangleq u_{1} V_{d c}, \quad$ and $i_{R} \triangleq u_{1} i_{q}+u_{2} i_{d}$

where, $u_{1} \triangleq \bar{u}_{g q}, u_{2} \triangleq \bar{u}_{g d}$, represent the average $(d-q)$ axis (Park's transformation) of the three-phase duty ratio system $\left(S_{1}, S_{2}, S_{3}\right)$, with:

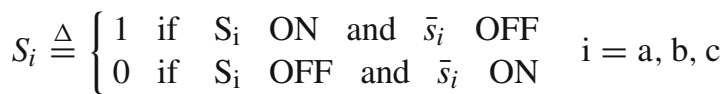

The input voltage between phases (line voltage) can be written in terms of switching action $S_{i}$ and $V_{d c}$, assuming that the generator side converter system is balanced without needing neutral point, such that:

$V_{g a b} \triangleq\left(S_{a}-S_{b}\right) V_{d c}$

$V_{g b c} \triangleq\left(S_{b}-S_{c}\right) V_{d c}$

$V_{g c a} \triangleq\left(S_{c}-S_{a}\right) V_{d c}$

Now, the following state space representation of the association (PMSG-rectifier) is given:

$$
\left\{\begin{array}{l}
\frac{d i_{d}}{d t}=-\frac{R_{a}}{L_{a}} i_{d}+n_{p} \omega_{g} i_{d}-i_{q} \omega_{g}-\frac{1}{L_{a}} u_{2} V_{d c} \\
\frac{d i_{q}}{d t}=-\frac{R_{a}}{L_{a}} i_{q}+n_{p} \omega_{g} i_{d}-\frac{\psi_{P M}}{L_{a}} \omega_{g}-n_{p} \omega_{g} i_{d}-\frac{1}{L_{a}} u_{1} V_{d c} \\
\frac{d \omega_{g}}{d t}=-1.5 \frac{n_{p}}{J} \psi_{P M} i_{q}-\frac{f}{J} \omega_{g}+\frac{1}{J} T_{g}
\end{array}\right.
$$

\subsection{Modeling of DC/AC converter}

The (DC/AC) PWM inverter circuit is shown in the right part of Fig. 2. The voltage stabilizer is connected to a converter, which consists of a three-phase converter with 6 semiconductors (IGBTs) with anti-parallel freewheeling diodes, for bidirectional current flow mode, displayed in three legs 1, 2, and 3 . The 6 semiconductors are considered as ideal switches. Only one switch on the same leg can be conducting at the same time. In order to model the DC bus voltage variation as a function of currents injected from the generator side converter and of those extracted on the grid side converter, the mathematical model is based on the following phase equations: 


$$
\begin{aligned}
& \left(L_{\text {inv }}+L_{\text {grid }}\right) \frac{d}{d t}\left[i_{L 123}\right] \\
& =\left[e_{L 123}\right]-V_{i n v}\left[G_{123}\right]-\left[i_{L 123}\right]\left(R_{i n v}+R_{\text {grid }}\right) \\
& -\frac{1}{C_{f i l}} \int i(t) d t \\
& 2 C_{\text {bus }} \frac{d V_{C_{\text {bus }}}}{d t}=i_{R}-i_{\text {inv }} \\
& i_{\text {inv }}=\left[G_{123}\right]^{T}\left[i_{L 123}\right] \\
& {\left[i_{\text {Linv } 123}\right]=\left[\frac{3}{\frac{e_{L 123}}{2}-V_{r m s} \sqrt{2}} i_{R}\right]} \\
& \sqrt{3} V_{d c} \\
& i_{R}=\frac{\sqrt{P^{2}+Q^{2}}}{V_{d c}}
\end{aligned}
$$

where, $\left[i_{L_{i n v}} 123\right]=\operatorname{col}\left[i_{L_{\text {inv }} 1}, i_{L_{\text {inv }} 2}, i_{L_{i n v} 3}\right]$, is the input current in the utility, $\left[e_{L_{i n v} 123}\right]=\operatorname{col}\left[e_{L_{i n v} 1,} e_{L_{i n v} 2}, e_{L_{i n v}}\right]$ is the sinusoidal three-phase voltages with known constant frequency $\omega_{n}, V_{d c}$ denotes the voltage across pair of capacitors $2 C_{\text {bus }}, i_{i n v}$ designates the input current inverter, and $G_{i}$ is the switch position function taking values in the discrete set $\{0,1\}$.

$G_{i} \triangleq\left\{\begin{array}{ccccccc}1 & \text { if } & \mathrm{G}_{\mathrm{i}} & \text { ON } & \text { and } & \overline{\mathrm{G}}_{1} & \text { OFF } \\ 0 & \text { if } & \mathrm{G}_{\mathrm{i}} & \text { OFF } & \text { and } & \overline{\mathrm{G}}_{1} & \text { ON }\end{array} \quad \mathrm{i}=\mathrm{a}, \mathrm{b}, \mathrm{c}\right.$

To simplify the mathematical representation of (6) for synthesis the third control law as follows:

$$
\left\{\begin{array}{c}
\frac{d}{d t} i_{L_{i n v}}=\frac{1}{\left(L_{\text {inv }}+L_{\text {grid }}\right)}\left[e_{L}-u_{3} V_{d c}\right. \\
\left.\quad-\left(R_{\text {inv }}+R_{\text {grid }}\right) i_{L_{i n v}}-\frac{1}{C_{f i l}} \int i(t) d t\right] \\
2 C_{\text {bus }} \frac{d V_{C_{\text {bus }}}}{d t}=i_{R}-i_{\text {inv }}
\end{array}\right.
$$

where, $u_{3}$ is the input switching control action of the inverter. The electrical grid is modeled using three sinusoidal signals connected to LCL filter output, while voltage signals from the grid side converter are sent to the input of the filter. The power absorbed by the DC/AC converter is given by the well-known expression is $P_{\text {absorbed }} \triangleq i_{i n v} V_{d c}$. on other hand, the power released to the AC load is given by, $P_{\text {released }} \triangleq e_{L_{i n v}} i_{L_{i n v}}$. Using the power conversion principle, one has, $P_{\text {absorbed }}=$ $P_{\text {released }}$, or equivalently, $i_{i n v} V_{d c}:=e_{L_{i n v}} i_{L_{i n v}}$. The cut-off frequency of the filter must be minimally ten times greater than grid frequency and simultaneously maximally one half of the converter switching frequency, because the filter must have enough attenuation in the range of the converter's switching frequency. The cut-off frequency must have a sufficient distance from the grid frequency. Finally, the whole nonlinear system model can be presented as given in [24].

As a matter of fact, the major difference between the model (8) and the model given in [24] resides in direct drive wind turbine generator system coupled with power grid via LCL grid filter. Unlike of the system model introduced by [24] and
[26], the mathematical model in those references is based on pure inductive filter. The inverter DC/AC output is tied to the electrical grid via an inductive filter that performs the filtering of current harmonics due to the inverter switching actions. A coupling LCL grid filter is used in present study to integrate the GSC side with electric grid. Definitely, with the LCL filters, the switching ripple components in grid currents will be decreased, drastically compared with same inductance value of the inductive-type filter while the dynamic performance is maintained.

$$
\Sigma_{d-q}\left\{\begin{array}{l}
\frac{d i_{d}}{d t}=-\frac{R_{a}}{L_{a}} i_{d}+n_{p} i_{q} \omega_{g}-\frac{1}{L_{a}} u_{2} V_{d c} \\
\frac{d i_{q}}{d t}=-\frac{R_{a}}{L_{a}} i_{q}+n_{p} \frac{\psi_{P M}}{L_{a}} \omega_{g}-n_{p} \omega_{g} i_{d}-\frac{1}{L_{a}} u_{1} V_{d c} \\
\frac{d \omega_{g}}{d t}=-1.5 \frac{n_{p}}{J} \psi_{P M} i_{q}-\frac{f}{J} \omega_{g}+\frac{1}{J} T_{g} \\
\dot{V_{d c}^{2}}=-\frac{1}{2 C_{b u s}} E_{n d} i_{n d}-\frac{1}{2 C_{b u s}}\left(E_{n q} i_{n q}-\mathrm{V}_{\mathrm{dc}} i_{R}\right) \\
\left.\dot{\iota_{n}}=-\frac{1}{L_{i n v}} E_{n d}+\omega_{\mathrm{s}} i_{n q}+\frac{1}{L_{i n v}} V_{d c} u_{3}\right) \\
\left.\dot{\iota_{n}}=-\frac{1}{L_{i n v}} E_{n q}+\omega_{\mathrm{s}} i_{n d}+\frac{1}{L_{i n v}} V_{d c} u_{4}\right)
\end{array}\right.
$$

\section{State feedback nonlinear controller design objectives}

\subsection{State feedback control objectives}

Backstepping control provides a systematic method to perform the design of a controller for nonlinear system. The main idea is to evaluate a control law in order to guarantee a positive definite and a negative time derivative Lyapunov function. The method is to split the system into a set of nested subsystems descending. The calculation of the Lyapunov function is performed.

The Control procedure is calculated in several steps and ensures the overall system stability [27]. The foundation of backstepping is the identification of a virtual control variable and forcing it to become a stabilizing function. Thus, it generates a corresponding error variable which can be stabilized by proper selection input via Lyapunov stability tools. One has four operational control objectives with four command input signals are named $u_{1}, u_{2}, u_{3}$, and, $u_{4}$ or by definition, $\bar{u}_{g q}, \bar{u}_{g d}, u_{n 1}$, and $u_{n 2}$, respectively.

C01: Speed Regulation: The generator rotor angular speed $\omega_{g}$ must track as given optimized signal, $\omega_{\text {opt }}$. This best value has been obtained from MPPT command strategy used in order to achieve best Tip Speed Ratio, TSR working conditions of the wind turbine to capture the maximum energy from the wind source [26].

CO2: Regulating the current $i_{d}$ to track a reference value $i_{d r e f}$. It is better to set it to zero in order to guarantee the absence of d-axis stator current, this implies no reluctance 
torque. Only the $q$-axis reactance is involved in producing the final voltage, i.e., there is no direct magnetization or demagnetization of $\mathrm{d}$-axis, only the field windings contribute to produce the flux along this direction [28].

CO3: Ensuring a satisfactory power factor correction (PFC) at the (grid-DDWTG) connection. The inverter output currents $\left(i_{L i n v 1}, i_{L i n v 2,} i_{L i n v 3}\right)$ must be sinusoidal with the same frequency.

CO4: Ensuring a tight regulation of the DC output voltage despite of wind acceleration and turbulence.

\subsection{State feedback speed regulation design}

For each wind velocity, there is best TSR that keeps the power coefficient at its best value. In order to achieve the best nominal TSR, it is required to control rotor speed follows best rotor speed, which can be produced by either measuring or estimating wind velocity. The speed regulator design is based on second and third equation of system (3), where the input control signal $u_{s q}$ represents the q-axis actual input, using the following backstepping design technique [29] to ensure an accurate optimized rotor speed tracking that leads us to optimize the capture wind energy equivalent to reference rotor speed.

Step 1: Let us define the following generator rotor angular speed tracking error is $\varepsilon_{\boldsymbol{\omega}} \triangleq \omega_{g}-\omega_{\text {opt }}$

In view of third equation of system (3), the above error will submit to the following equation:

$$
\begin{aligned}
\dot{\varepsilon_{\omega}}= & \frac{\mathrm{d} \omega_{\mathrm{g}}}{\mathrm{dt}}-\dot{\omega}_{o p t}=-1.5 \frac{n_{p}}{J} \psi_{\mathrm{PM}} i_{q}-\frac{f}{J} \omega_{g} \\
& +\frac{1}{J} T_{g}-\omega_{o p t}^{\dot{*}}
\end{aligned}
$$

According to (9), the design parameter: $\rho=-1.5 \frac{n_{p}}{J} \psi_{\mathrm{PM}} i_{q}$, stands up as a virtual control input for first error dynamics, $\dot{\varepsilon_{\omega}}$.

Let us consider $\rho_{\text {ref }}$ denotes the stabilizing function concerning to $\rho$. It is readily observed from (9) that, if $\rho=\rho_{\text {ref }}$ with:

$\rho_{\text {ref }} \triangleq-h_{1} \varepsilon_{\omega}+\frac{f}{J} \omega_{g}-\frac{1}{J} T_{g}+\varepsilon_{\text {opt }}^{\cdot}$

with $h_{1}$ is positive design parameter. As a matter of fact, if $\rho=\rho_{\text {ref }}$, one has $\dot{\varepsilon_{\omega}}=-h_{1} \varepsilon_{\boldsymbol{\omega}}$, which readily is asymptotically stable with respect to the Lyapunov function:

$W_{1}\left(\varepsilon_{\omega}\right) \triangleq \frac{1}{2} \varepsilon_{\omega}^{2}$

Then take the time derivative along (8), yields

$$
\dot{W}_{1}\left(\varepsilon_{\omega}\right)=-\varepsilon_{\omega} \dot{\varepsilon_{\omega}}<0
$$

As, $\rho=-1.5 \frac{n_{p}}{J} \psi_{\mathrm{PM}} i_{q}$ is just a virtual control input, one cannot consider, $\rho=\rho_{\text {ref }}$.

However, the above expression of $\rho_{\text {ref }}$ is considered as first stabilizing function and a new error is presented by:

$\varepsilon_{q} \triangleq \rho-\rho_{\text {ref }}=-1.5 \frac{n_{p}}{J} \psi_{\mathrm{PM}} i_{q}+1.5 \frac{n_{p}}{J} \psi_{\mathrm{PM}} i_{q r e f}$

$\varepsilon_{q} \triangleq\left(i_{\text {qref }}-i_{q}\right) \frac{3 n_{p}}{J} \psi_{\mathrm{PM}}$

Using systems (10)-(13), it follows from (9) the first error dynamics is, $\dot{\varepsilon_{\omega}}$ :

$\dot{\varepsilon_{\omega}}=-h_{1} \varepsilon_{\omega}+\varepsilon_{q}$

Step 2: The next step composite of determining the first control input $u_{1}$, such that the error system $\left(\varepsilon_{\boldsymbol{\omega}}, \varepsilon_{q}\right)$ is asymptotically stable. Let us get the trajectory of error, $\varepsilon_{q}$. The time derivative $\varepsilon_{q}$ along the closed-loop trajectory of (13):

$\dot{\varepsilon_{q}}=\dot{\rho}-\rho_{\text {ref }}=-1.5 \frac{n_{p}}{J} \psi_{\mathrm{PM}} i_{q}+1.5 \frac{n_{p}}{J} \psi_{\mathrm{PM}} i_{q_{r e f}}$

Substituting the right hand side of (8), with aid of second and third equation of system (5) in (15), one can obtain:

$\dot{\varepsilon_{q}}=\xi\left(i_{d}, i_{q}, \omega_{g}\right)-h_{1}^{2} \varepsilon_{\boldsymbol{\omega}}+h_{1} \varepsilon_{q}+\frac{n_{p}}{J} \psi_{\mathrm{PM}} u_{1} \mathrm{~V}_{\mathrm{dc}}$

with, $\xi\left(i_{d}, i_{q}, \omega_{g}\right)$ is a nonlinear function defined in form:

$$
\begin{gathered}
\xi\left(i_{d}, i_{q}, \omega_{g}\right) \triangleq \frac{n_{p}}{J} \psi_{\mathrm{PM}}\left(1.5 \frac{R_{a}}{L_{a}} i_{q}+n_{p} \omega_{g} i_{d}-\frac{n_{p}}{L_{a}} \psi_{\mathrm{PM}} \omega_{g}\right) \\
+\left(\frac{f}{J}\right)^{2} \omega_{g}+\frac{f n_{p}}{J^{2}} \psi_{\mathrm{PM}} i_{q}-\frac{f T_{g}}{J^{2}}+\frac{1}{J} \dot{T}_{g}-\ddot{\rho_{\text {ref }}}
\end{gathered}
$$

The error dynamics (14) and (16) are given the compact form:

$$
\begin{aligned}
& \dot{\varepsilon_{\boldsymbol{\omega}}}=-h_{1} \varepsilon_{\boldsymbol{\omega}}+\varepsilon_{q} \\
& \dot{\varepsilon_{q}}=\xi\left(i_{d}, i_{q}, \omega_{g}\right)-h_{1}^{2} \varepsilon_{\boldsymbol{\omega}}+h_{1} \varepsilon_{q}+\frac{n_{p}}{J} \psi_{\mathrm{PM}} u_{1} \mathrm{~V}_{\mathrm{dc}}
\end{aligned}
$$

To compute stabilization control law for (18b); let us define the quadratic Lyapunov function candidate:

$W_{2}\left(\varepsilon_{\boldsymbol{\omega}}, \varepsilon_{q}\right) \triangleq W_{3}\left(\varepsilon_{\boldsymbol{\omega}}\right)+\frac{1}{2} \varepsilon_{\omega}^{2}$

In view of (12), the time derivative of $W_{2}\left(\varepsilon_{\boldsymbol{\omega}}, \varepsilon_{q}\right)$ along the trajectories can be rewritten as:

$\dot{W}_{2}\left(\varepsilon_{\boldsymbol{\omega}}, \varepsilon_{q}\right)=-h_{1} \varepsilon_{\boldsymbol{\omega}}^{2}+\varepsilon_{\boldsymbol{\omega}} \varepsilon_{q}+\varepsilon_{q} \dot{\varepsilon_{q}}$ 
This illustrates that for the system error $\left(\varepsilon_{\boldsymbol{\omega}}, \varepsilon_{q}\right)$ to be global asymptotically stable GAS, it is sufficient to choose the control input signal $u_{1}$, such that:

$$
\dot{W}_{2}\left(\varepsilon_{\omega}, \varepsilon_{q}\right)=-h_{1} \varepsilon_{\omega}^{2}-h_{2} \varepsilon_{q}^{2}
$$

with $h_{2}$ is a new positive design parameter. Using (20b), equation (20a) is guaranteed if:

$\dot{\varepsilon_{q}}=-h_{2} \varepsilon_{q}-\varepsilon_{\omega}$

Comparing (21) and second subsystem of (18) yields the following average generator q-axis of the three-phase (duty cycle) system $\left(S_{1}, S_{2}, S_{3}\right)$ :

$$
\begin{aligned}
u_{1}= & \frac{J}{n_{p} \psi_{\mathrm{PM}}}\left[-\left(h_{1}+h_{2}\right) \varepsilon_{q}+\left(h_{1}^{2}-1\right)\right. \\
& \left.\varepsilon_{\omega}-\xi\left(i_{d}, i_{q}, \omega_{g}\right)\right] / \mathrm{V}_{\mathrm{dc}}
\end{aligned}
$$

Let us define, $u_{1} \triangleq \bar{u}_{\mathrm{gq}}$, which represents the average q-axis system duty cycle:

$u_{g q}=\bar{u}_{g q} V_{d c}$

Remark 1 Indeed, it is easily checked that the denominator of (22) never vanish (never tends to zero) in practice because of the generator residual flux (remnant flux in rotor).

\subsection{Direct-axis current regulation design}

Step 3: The direct-axis stator current $i_{d}$ stated in system (32) such that the following quantity is presented at steady state condition:

$\Lambda\left(\mathrm{i}_{\mathrm{q}}, \omega_{\mathrm{g}}, \mathrm{V}_{\mathrm{dc}}\right) \triangleq n_{p} \mathrm{i}_{\mathrm{q}} \omega_{\mathrm{g}}-\frac{1}{\mathrm{~L}_{\mathrm{a}}} u_{2} \mathrm{~V}_{\mathrm{dc}}$

The ideal machine has zero resistance and leakage reactance, infinite permeability, and no saturation, as well as zero reluctance torque. To ensure that the reluctance torque $T_{R}$ is omitted, $T_{g}=T_{P M}+T_{R}=\frac{3 n_{p}}{2}\left[\psi_{P M} i_{q}+\left(L_{d}-L_{q}\right) i_{d} i_{q}\right]$, where first and second term represents permanent torque and reluctance torque, respectively. This decouples the torque and flux providing faster transient response, which makes the control task easier. The reference direct axis current $i_{d}^{\text {ref }}$ must be equal zero, it follows that the new tracking error is:

$\varepsilon_{d} \triangleq i_{d}-i_{d}^{r e f}=i_{d}$

The time derivative of $\varepsilon_{d}$ is:

$\dot{\varepsilon_{d}}=\frac{1}{k t} \varepsilon_{\omega}+\Lambda\left(\mathrm{i}_{\mathrm{q}}, \omega_{\mathrm{g}}, \mathrm{V}_{\mathrm{dc}}\right)$ with, $k t=\frac{L_{a}}{R_{a}}$ is the electrical time constant. To get the second stabilizing control signal for system (26), let us define the following quadratic Lyapunov function:

$W_{3}\left(\varepsilon_{d}\right) \triangleq \frac{1}{2} \varepsilon_{d}^{2}$

It is readily checked, if the virtual control signal is to be:

$\Lambda\left(\mathrm{i}_{\mathrm{q}}, \omega_{\mathrm{g}}, \mathrm{V}_{\mathrm{dc}}\right) \triangleq-\left(\frac{1}{K_{t}}+h_{3}\right) \varepsilon_{d}$,

with $h_{3}$ is positive design parameter. Substituting the right hand side of (28) in (25), one gets third error:

$\dot{\varepsilon_{d}}=\left(\frac{1}{K_{t}} \varepsilon_{d}-\frac{1}{K_{t}} \varepsilon_{\omega}-h_{3} \varepsilon_{d}\right)=-h_{3} \varepsilon_{d}$

Thus,

$\dot{W}_{3}\left(e_{d}\right)=\varepsilon_{d} \dot{\varepsilon_{d}}=\varepsilon_{d}\left(-h_{3} \varepsilon_{d}\right)=-h_{3} \varepsilon_{d}^{2}<0$

So, it is easily checked that the actual control input signal is obtained by substituting (28) in (24) and after simple mathematical manipulation, one gets:

$$
\begin{aligned}
\frac{1}{K_{t}} \varepsilon_{d}-h_{3} \varepsilon_{d} & =n_{p} \mathrm{i}_{\mathrm{q}} \omega_{\mathrm{g}}-\frac{1}{\mathrm{~L}_{\mathrm{a}}} \mathrm{u}_{2} \mathrm{~V}_{\mathrm{dc}} \\
\text { Or, } \quad u_{2} & =\frac{\mathrm{L}_{\mathrm{a}}}{\mathrm{V}_{\mathrm{dc}}}\left[n_{p} \mathrm{i}_{\mathrm{q}} \omega_{\mathrm{g}}-\frac{1}{K_{t}} \varepsilon_{d}+h_{3} \varepsilon_{d}\right] \triangleq \bar{u}_{\mathrm{gd}}
\end{aligned}
$$

It represents the average direct- and q-axis.

$u_{g d}=\bar{u}_{g d} V_{d c}$

Let us define state feedback practical control law in $(\alpha-\beta)$ coordinates as,

$$
\begin{aligned}
& {\left[\begin{array}{l}
u_{\alpha} \\
u_{\beta}
\end{array}\right]=\left[\begin{array}{cc}
\cos \hat{\theta}_{r e} & -\sin \hat{\theta}_{r e} \\
\sin \hat{\theta}_{r e} & \cos \hat{\theta}_{r e}
\end{array}\right]} \\
& {\left[\begin{array}{l}
u_{c d} \\
u_{c q}
\end{array}\right]=u_{S F C} \triangleq \zeta\left(T_{g}, \omega_{g}, i_{q}, i_{d}\right)}
\end{aligned}
$$

Finally, PWM concept is used in order to produce the control signal to implement the nonlinear control for the PMSG. The control voltages $\mathrm{u}_{1}$ and $\mathrm{u}_{2}$ are calculated using equation (22) and (31); they are converted to three-phase voltages using Park's transformation.

\section{DC voltage controller and reactive power}

In controlling a power factor correction, the main objective is to obtain a sinusoidal output current and the injection 
or extraction of a desired reactive power in the electric network. The continuous voltage $V_{d c}$ must track a given reference signal $V_{d c r e f}$. These objectives lead to two control loops. The first loop ensures the regulation of the DC voltage and the second ensures the injection of the desired reactive power.

Based on system (8), the equation involving the control input $u_{3}$ will now be designed, using the backstepping technique, so that the squared DC-link voltage follows well any reference signal $V_{d c r e f}>0$. As the system (5) is relative degree 2 , the design towards that equation is performed in two steps.

\subsection{Voltage controller}

\section{Step 1. DC link voltage tracking error}

Let $\varepsilon_{V d c}$ denote the DC link voltage tracking error:

$\varepsilon_{V d c}=V_{d c}^{2}-V_{d c r e f}^{2}$

The problem at hand is to design of third control law $u_{3}$ such that the DC squared voltage, $V_{d c}^{2}$ tracks a given reference signal, $V_{d c r e f}^{2}$ where, $V_{d c r e f}$ is a desired reference signal must be greater than, $2 * V_{g n}$, to ensure the occurance elevation feature of the boost power converter. In view of (8d), the above error submits to the following dynamic error:

$$
\begin{aligned}
& \dot{\varepsilon}_{V d c}=-\frac{1}{2 C_{b u s}} E_{n d} i_{n d} \\
&+\Upsilon\left(\mathrm{i}_{\mathrm{d}}, \mathrm{i}_{\mathrm{q}}, \omega_{\mathrm{g}}, V_{d c}^{2}, i_{n d} i_{n q}, \varepsilon_{\omega}, \varepsilon_{q}, \varepsilon_{d}\right) \\
&-V_{d c r e f}^{2} \Upsilon\left(\mathrm{i}_{\mathrm{d}}, \mathrm{i}_{\mathrm{q}}, \omega_{\mathrm{g}}, V_{d c}^{2}, i_{n d} i_{n q}, \varepsilon_{\omega}, \varepsilon_{q}, \varepsilon_{d}\right) \\
& \dot{\varepsilon}_{V d c}=-\frac{1}{2 C_{b u s}} E_{n q} i_{n q}+\frac{J \mathrm{~L}_{\mathrm{a}}}{3 n_{p} \psi_{\mathrm{PM}} \sqrt{3 / 2}} \\
&\left\{\left(h_{1}+h_{2}\right) \varepsilon_{q}-h_{1}^{2} \varepsilon_{\omega}+\mathrm{\Psi}\left(\mathrm{i}_{\mathrm{d}}, \mathrm{i}_{\mathrm{q}}, \omega_{\mathrm{g}}, V_{d c}^{2}, i_{n d} i_{n q}, t\right) \mathrm{i}_{\mathrm{q}}\right. \\
&\left.-\mathrm{L}_{\mathrm{a}}\left(h_{3} \varepsilon_{d}-\frac{R_{a}}{L_{a}} \varepsilon_{d}+n_{p} \mathrm{i}_{\mathrm{d}} \omega_{\mathrm{g}}\right) \mathrm{i}_{\mathrm{d}}\right\}
\end{aligned}
$$

In (35), the magnitude $\rho_{2}=-\frac{1}{2 C_{b u s}} E_{n d} i_{n d}$ stands up as second virtual control input for the $\dot{\varepsilon}_{V d c}$ dynamics because the third actual control input $u_{3}$ effects on $\varepsilon_{V d c}$ indirectly through $\rho_{2}$. Following the nonlinear backstepping design technique, the Lyapunov function candidate is considered as:

$W_{4}=\frac{1}{2} \varepsilon_{V d c}^{2}$

Deriving $W_{4}$ along the closed-loop trajectory of (35), yields:

$$
\begin{aligned}
\dot{W}_{4}= & \varepsilon_{V d c} \dot{\varepsilon}_{V d c} \\
= & -\varepsilon_{V d c}\left(-\frac{1}{2 C_{b u s}} E_{n d} i_{n d}\right. \\
& \left.-\Upsilon\left(\mathrm{i}_{\mathrm{d}}, \mathrm{i}_{\mathrm{q}}, \omega_{\mathrm{g}}, V_{d c}^{2}, i_{n d} i_{n q}, \varepsilon_{\omega}, \varepsilon_{q}, \varepsilon_{d}\right)+V_{d c r e f}^{2}\right)
\end{aligned}
$$

This puts for the second virtual control law $\rho_{2}$ the following control law as follows:

$$
\begin{aligned}
\rho_{2}^{r e f}= & -h_{4} \varepsilon_{V d c} \\
& -\Upsilon\left(\mathrm{i}_{\mathrm{d}}, \mathrm{i}_{\mathrm{q}}, \omega_{\mathrm{g}}, V_{d c}^{2}, i_{n d} i_{n q}, \varepsilon_{\omega}, \varepsilon_{q}, \varepsilon_{d}\right) \\
& +V_{\text {dcref }}^{2}
\end{aligned}
$$

where $h_{4}$ is positive design parameter, $h_{4}>0$. So, backsubstituting $\rho_{2}^{r e f}$ to $\rho_{2}=-\frac{1}{2 C_{b u s}} E_{n d} i_{n d}$, it will give, $\dot{W}_{4}=$ $-h_{4} \varepsilon_{V d c}^{2}$, which ensures negative definite in $\varepsilon_{V d c}$. As $\rho_{2}$ is only a virtual control input, one cannot set $\rho_{2}=\rho_{2}^{\text {ref }}$. Still the above expression of $\rho_{2}^{r e f}$ is retained and a new error is introduced as:

$\varepsilon_{n d}=\rho_{2}-\rho_{2}^{r e f}$

Using (38), it follows from (35) that the $\dot{\varepsilon}_{V d c}$ dynamics undergoes the following equation:

$\dot{\varepsilon}_{V d c}=-h_{4} \varepsilon_{V d c}+\varepsilon_{n d}$

Step 2. Now, the aim is to make the couple of errors $\left(\varepsilon_{V d c}, \varepsilon_{n d}\right)$ null asymptotically. The closed-loop trajectory of the error $e_{n d}$ is obtained by taking time derivation of (39) i.e.:

$$
\begin{aligned}
\dot{\varepsilon}_{n d}= & -\frac{1}{2 C_{b u s}} E_{n d} i_{n d}+\dot{\Upsilon} \\
& \times\left(\mathrm{i}_{\mathrm{d}}, \mathrm{i}_{\mathrm{q}}, \omega_{\mathrm{g}}, V_{d c}^{2}, i_{n d}, i_{n q}, \varepsilon_{\omega}, \varepsilon_{q}, \varepsilon_{d}\right) \\
& +h_{4} \dot{\varepsilon}_{V d c}+\ddot{V}_{d c r e f}^{2}
\end{aligned}
$$

Using (40) and (8d)-(8e) combined in (41), yields:

$$
\begin{aligned}
\dot{\varepsilon}_{n d}= & \Upsilon_{1}\left(\mathrm{i}_{\mathrm{d}}, \mathrm{i}_{\mathrm{q}}, \omega_{\mathrm{g}}, V_{d c}^{2}, i_{n d}, i_{n q}, \varepsilon_{\omega}, \varepsilon_{q}, \varepsilon_{d}, \varepsilon_{V d c}, \varepsilon_{n d}\right) \\
& -\frac{1}{2 C_{b u s} L_{i n v}} E_{n d} u_{3} V_{d c}
\end{aligned}
$$

with

$$
\begin{aligned}
& \Upsilon_{1}\left(\mathrm{i}_{\mathrm{d}}, \mathrm{i}_{\mathrm{q}}, \omega_{\mathrm{g}}, V_{d c}^{2}, i_{n d}, i_{n q}, \varepsilon_{\omega}, \varepsilon_{q}, \varepsilon_{d}, \varepsilon_{V d c}, \varepsilon_{n d}\right) \\
& \triangleq-h_{4} \varepsilon_{V d c}
\end{aligned}
$$




$$
\begin{aligned}
& +\dot{\Upsilon}\left(\mathrm{i}_{\mathrm{d}}, \mathrm{i}_{\mathrm{q}}, \omega_{\mathrm{g}}, V_{d c}^{2}, i_{n d}, i_{n q}, \varepsilon_{\omega}, \varepsilon_{q}, \varepsilon_{d}\right) \\
& -\ddot{V}_{d c r e f}^{2}+\frac{E_{n d}^{2}}{2 C_{b u s} L_{i n v}}-\frac{E_{n d}}{2 C_{b u s}} \omega_{\mathrm{s}} i_{n q}
\end{aligned}
$$

To determine a stabilizing control law for (8d)-(8e), let us consider the quadratic Lyapunov function candidate:

$W_{5}=\frac{1}{2} \varepsilon_{V d c}^{2}+\frac{1}{2} \varepsilon_{n d}^{2}$

Using (40)-(42), one gets from (43) the time derivative of fifth Lyapunov function that is:

$$
\begin{aligned}
\dot{W}_{5}= & \varepsilon_{V d c} \dot{\varepsilon}_{V d c}+\varepsilon_{n d} \dot{\varepsilon}_{n d} \\
\dot{W}_{5}= & -h_{4} \varepsilon_{V d c}^{2}+\varepsilon_{n d}\left\{\varepsilon_{V d c}\right. \\
& +\Upsilon_{1}\left(\mathrm{i}_{\mathrm{d}}, \mathrm{i}_{\mathrm{q}}, \omega_{\mathrm{g}}, V_{d c}^{2}, i_{n d}, i_{n q}, \varepsilon_{\omega}, \varepsilon_{q}, \varepsilon_{d}, \varepsilon_{V d c}, \varepsilon_{n d}\right) \\
& \left.-\frac{1}{2 C_{b u s} L_{i n v}} u_{3} V_{d c}\right\}
\end{aligned}
$$

This gives us the third practical control law $u_{3}$ as:

$$
\begin{aligned}
& u_{3}=\left\{h_{5} \varepsilon_{n d}+\varepsilon_{V d c}\right. \\
&\left.+\Upsilon_{1}\left(\mathrm{i}_{\mathrm{d}}, \mathrm{i}_{\mathrm{q}}, \omega_{\mathrm{g}}, V_{d c}^{2}, i_{n d}, i_{n q}, \varepsilon_{\omega}, \varepsilon_{q}, \varepsilon_{d}, \varepsilon_{V d c}, \varepsilon_{n d}\right)\right\} \\
& \frac{2 C_{b u s} L_{i n v}}{E_{n d} V_{d c}}
\end{aligned}
$$

with $h_{5}$ is a positive design parameter, $h_{5}>0$. So, substituting (45) in (44), yields:

$\dot{W}_{5}=-h_{4} \varepsilon_{V d c}^{2}-h_{5} \varepsilon_{n d}^{2}<0$

Now, substituting (45) in (42) one obtains the DC voltage closed-loop control system:

$\dot{\varepsilon}_{V d c}=-h_{4} \varepsilon_{V d c}+\varepsilon_{n d}, \dot{\varepsilon}_{n d}=-h_{5} \varepsilon_{n d}-\varepsilon_{V d c}$

\subsection{Reactive power control}

This study shall pay attention to the control objective $\mathbf{C O}_{3}$ that involves the network reactive power, $Q_{n}$, which is required to track its reference $Q_{n r e f}$. The electrical reactive power injected in the electrical network is given by $Q_{n} \triangleq E_{n d} i_{n q}-E_{n q} i_{n d}$. To normalize notation throughout this section, the corresponding tracking error is denoted as, $\varepsilon_{n q} \triangleq Q_{n}-Q_{n r e f}$. It follows from (8e-8f) that $\varepsilon_{n q}$ submits to the following differential equation: $\dot{\varepsilon}_{n q}=\Upsilon_{2}\left(i_{n d}, i_{n q}\right)+\frac{\mathrm{V}_{\mathrm{dc}}}{L_{i n v}}\left(E_{n d} u_{4}-E_{n q} u_{3}\right)$

with, $\Upsilon_{2}\left(i_{n d}, i_{n q}\right) \triangleq-\omega_{\mathrm{s}}\left(E_{n d} i_{n d}+E_{n q} i_{n q}\right)-\dot{Q}_{n r e f}$

As the Eq. (48) is first order differential equation, it can be globally asymptotically stabilized using a simple proportional control law with, $h_{6}>0$ :

$\frac{\mathrm{V}_{\mathrm{dc}}}{L_{i n v}}\left(E_{n d} u_{4}-E_{n q} u_{3}\right)=-h_{6} \varepsilon_{n q}-\Upsilon_{2}\left(i_{n d}, i_{n q}\right)$

Then the fourth practical control law $\mathrm{u}_{4}$ is given as:

$u_{4}=\frac{-L_{i n v}\left(h_{6} \varepsilon_{n q}+\Upsilon_{2}\left(i_{n d}, i_{n q}\right)\right.}{E_{n d}}$

It can be easily checked that the dynamic of $\varepsilon_{n q}$ submits to the following equation:

$\dot{\varepsilon}_{n q}=-h_{6} \varepsilon_{n q}$

The control closed-loops induced by the DC link voltage and reactive power practical control laws thus, defined by (45) and (50) are analysed and discussed successfully.

\section{Semi-global output feedback controller design}

The controller developed in section three has been formally shown to achieve all the control objectives listed in Sect. 3.1. The point is that this controller was designed using the $(d-q)$ model which necessitates for online measurements of several state variables including the rotor position. As there are no cheap and unreliable sensors of these variables, the above controller will remain useless. So, an observer is developed providing accurate estimates of the non-measurement variables. The main purpose of the present section is to design sampled high-gain observer and use it to build up an output feedback controller that does not need measuring the optimized wind velocity, rotor speed and generator torque.

\subsection{Modeling of PMSG in $(\alpha-\beta)$ reference frame}

Modeling is a basic tool for analysis, such as optimization, project, design and control. Wind energy conversion systems are very different in nature from conventional generators, and therefore dynamic studies must be addressed in order to integrate wind power into the power system. Models utilized for steady state analysis are extremely simple, while the dynamic models for wind energy conversion systems are not easy to develop. Dynamic modelling is needed for various types of analysis related to system dynamics: stability, control system, and optimization. 
The PMSG model is constructed in $(\boldsymbol{\alpha}-\boldsymbol{\beta})$ stationary reference frame, which is most suitable for sampled data state space observer design. The control objective is to determine under what sufficient conditions that all the PMSG states variables, which are $i_{g}, \psi_{r} \quad \omega_{\mathrm{g}}$ and $T_{g}$ can be determined from the generator input and output measurements, namely the stator current and the generator input command voltage signal, $\mathrm{i}_{\mathrm{g}}$ and $u_{g}$. The rotor reference frame $(\mathrm{d}-\mathrm{q})$ is generally used for its simplicity in nonlinear feedback controller design, which needs the initial rotor position detection before stating up the wind turbine generator DDWTG system. Introduced by $[27,28]$, the DDWTG system model is:

$\Sigma$ PMSG $\left\{\begin{array}{l}\frac{d i_{g}}{d t}=-\frac{R_{a}}{L_{a}} i_{g}-\frac{n_{p}}{L_{a}} \omega_{g} \quad T_{2} \psi_{r}-\frac{1}{L_{a}} u_{g} \\ \frac{d \psi_{r}}{d t}=n_{p} \omega_{g} T_{2} \psi_{r} \\ \frac{d \omega_{g}}{d t}=-\frac{3}{2} \frac{n_{p}}{J} i_{g}^{T} T_{2} \psi_{r}-\frac{f}{J} \omega_{g}+\frac{1}{J} T_{g} \\ \frac{d T_{g}}{d x} \approx 0\end{array}\right.$

with $i_{g} \triangleq\left[\begin{array}{ll}i_{g \alpha} & i_{g \beta}\end{array}\right]^{T}, \psi_{r} \triangleq\left[\begin{array}{ll}\psi_{r \alpha} & \psi_{r \beta}\end{array}\right]^{T}, u_{g} \triangleq\left[\begin{array}{ll}u_{g \alpha} & u_{g \beta}\end{array}\right]^{T}$ are respectively, the stator vector of currents, the rotor fluxes and the generator input command signals. $\omega_{\mathrm{g}}$ and $T_{g d}$ respectively, denote the generator rotor speed and the generator torque with influence of wind turbulence, which is unknown but bounded and that its upper bound is available. $T_{2}$ is the matrix $\in R^{2 \times 2}$ defined as follows: $\mathrm{T}_{2}=\left[\begin{array}{cc}0 & -1 \\ 1 & 0\end{array}\right] ; J$ and $f$ are the generator moment of inertia and viscous friction; $n_{p}$ is the number of pole pairs of. The electrical parameters, $R_{a}$ and $L_{a}$ are the armature resistor and inductance, respectively.

The (AC/DC/AC) power electronics converter is divided in two components: the generator side converter and the grid side converter [30].

Let us study the observability concept of system (52) by considering the stator current output measurement in $(\alpha-\beta)$ reference frame is $y \triangleq i_{g}$ as output vector. For sake of clarity, one can introduce the following state vectors:

$$
\begin{gathered}
x \triangleq\left(x_{1} x_{2} x_{3}\right)^{T}, \\
\left\{\begin{array}{l}
x_{1} \triangleq\left(x_{11}, x_{12}\right)^{T}=\left(i_{g \alpha}, i_{g \beta}\right)^{T} \\
x_{2} \triangleq\left(x_{21}, x_{22}\right)^{T}=\left(\omega_{g}, T_{g}\right)^{T} \\
x_{3} \triangleq\left(x_{31}, x_{32}\right)^{T}=\left(\psi_{r \alpha}, \psi_{r \beta}\right)^{T}
\end{array}\right.
\end{gathered}
$$

where $x_{1}, x_{2}$ and $x_{3}$ are the pair of distinct states. As a result, the notation $I_{k}$ and $0_{\mathrm{k}}$ will be used to denote $\mathrm{k} \times \mathrm{k}$ is identity matrix and the $\mathrm{k} \times \mathrm{k}$ is null matrix, respectively. The rectangular $(\mathrm{k} \times \mathrm{m})$ null matrix will be denoted by $0_{\mathrm{k} \times \mathrm{m}}$. System (1) can then be re-written under the following compact form for MIMO system: $\left\{\begin{array}{l}\dot{x}=f\left(x, u_{g}\right)+B_{c} T_{g} \\ y=C_{c} x(t)=x_{1}=h(x)\end{array}\right.$

where, the vector field function,

$$
\begin{aligned}
& f\left(x, u_{g}\right) \triangleq\left(\begin{array}{l}
f_{1}\left(x, u_{g}\right) \\
f_{2}\left(x, u_{g}\right) \\
f_{3}\left(x, u_{g}\right)
\end{array}\right) \\
& =\left(\begin{array}{l}
-\frac{n_{p}}{L_{s}} x_{21} T_{2} x_{3}-\frac{R_{s}}{L_{s}} x_{1}-\frac{1}{L_{s}} u_{g} \\
n_{p} x_{21} T_{2} x_{3} \\
\left\{\frac{-3 n_{p}}{2 J} x_{1}^{T} T_{2} x_{2}-\frac{f}{J} x_{31}+\frac{1}{J} x_{32}, 0\right\}
\end{array}\right)
\end{aligned}
$$

The nonlinear system matrices are: $B_{c} \triangleq\left[0_{5 \times 1} 1\right]^{T}, C_{c} \triangleq$ $\left[\mathrm{I}_{2} \mathrm{O}_{2} \mathrm{O}_{2}\right]$.

\subsection{Observability analysis of sensorless PMSG}

The proposed wind turbine generator system is not in normal form of observability concept. Thus, it is required define sufficient conditions such that the considered state transformation is globally diffeomorphic. The observation objective is to reconstruct the rotor speed, rotor flux assuming that they are unavailable by measurement and moreover under the fact that the armature winding resistance and armature winding inductance are known.

One can conclude that, the system (3) is observable for any generator input $u_{g}$. Consequently, it will be observable in the full rank as soon as the observability map $\Phi(\mathrm{x})$ exists and is regular almost everywhere. Notice that, we should find a sufficient condition under which the Jacobian matrix is full rank almost everywhere. From first subsystem model of (3) wind turbine generator in $(\alpha-\beta)$ reference frame, gives

$\left[\begin{array}{c}\frac{\mathrm{di}_{\mathrm{g} \alpha}}{\mathrm{dt}} \\ \frac{\mathrm{di}_{\mathrm{g} \beta}}{\mathrm{dt}}\end{array}\right]=-\frac{R_{a}}{L_{a}}\left[\begin{array}{c}\mathrm{i}_{\mathrm{g} \alpha} \\ \mathrm{i}_{\mathrm{g} \beta}\end{array}\right]+\frac{n_{p}}{L_{a}} \quad \omega_{m}\left[\begin{array}{c}\psi_{r \beta} \\ -\psi_{r \alpha}\end{array}\right]-\frac{1}{L_{a}}\left[\begin{array}{c}\mathrm{u}_{\mathrm{g} \alpha} \\ \mathrm{u}_{\mathrm{g} \beta}\end{array}\right]$

Let us denote: $h(x) \triangleq\left[h_{1}(x), h_{2}(x)\right]^{T}=\left[i_{g \alpha}, i_{g \beta}\right]^{T}$.

The property of observability can be evaluated from the measured state variables and their corresponding derivatives, respectively. Let us consider the following observation space containing the information that generated for the observability criterion knowing that $L_{f}^{k} h$ is called the $k^{\prime}$ th order Lie -derivative of the function $h(x)$ with respect to the vector field, $f\left(x, u_{g}\right)=\left[f_{1}\left(x, u_{g}\right), f_{2}\left(x, u_{g}\right), f_{3}\left(x, u_{g}\right)\right]^{T}$.

Lie-derivative of the function $h(x)$ along the vector field $f(x)$ is a new scalar function defined by $L_{f} h(x)$, which is obtained as: 


$$
\begin{aligned}
O_{W T G} h(x)= & \left\{h_{1}(x), h_{2}(x), L_{f} h_{1}(x),\right. \\
& \left.L_{f} h_{2}(x), L_{f}^{2} h_{1}(x), L_{f}^{2} h_{2}(x)\right\}
\end{aligned}
$$

Such that, $\left.h(x) \triangleq\left[L_{f}^{0} h_{1}(x), L_{f}^{0} h_{2}\right)(x)\right]^{T}=\left[h_{1}(x)\right.$, $\left.h_{2}(x)\right]^{T}$.

Then, the observability analysis of the WTG is made by verifying if the observability matrix is locally observable at $x_{0}$. It is obvious that the observability analysis is made by evaluating the Jacobian of nonlinear systems, $J_{O_{W T G}} h(x)$, with respect to all machine state variables $x$, where $x_{0} \in X \subset$ $R^{6}$ is a certain point from its state space representation.

$\left.J_{O_{W T G}} h(x) \triangleq \frac{\partial}{\partial x} O_{W T G} h(x)\right|_{x=x_{0}}$

The Jacobian matrix characterizes is the observability of the WTG model in the rank sense. If the $J_{O_{W T G}} h(x)$ has full rank, this means that, $\left.\operatorname{dim}\left(J_{O_{W T G}} h(x)\right)\right|_{x=x_{0}}=6$, so if the observability rank condition holds $\forall x \in R^{6}$, then system (54) is observable in the rank sense.

$$
\begin{aligned}
& \left.\operatorname{rank}\left\{J_{O_{W T G}} h(x)\right\}\right|_{x=x_{0}} \\
& =\operatorname{rank}\left(\begin{array}{c}
d h_{1}(x) \\
d h_{2}(x) \\
d L_{f} h_{1}(x) \\
d L_{f} h_{2}(x) \\
d L_{f}^{2} h_{1}(x) \\
d L_{f}^{2} h_{2}(x)
\end{array}\right)=n=6
\end{aligned}
$$

where $d$ is the usual partial derivative. The state of the system model (52) is observable. The associated observability matrix gives observability criterion matrix has dimensions of $(6 \times 6)$, is:

$$
\begin{aligned}
& J_{O_{W T G}} h(x) \\
& \triangleq\left[\begin{array}{cccccc}
1 & 0 & 0 & 0 & 0 & 0 \\
0 & 1 & 0 & 0 & 0 & 0 \\
\frac{\partial L_{f} \mathrm{~h}_{1}(x)}{\partial x_{11}(x)} & \frac{\partial L_{f} \mathrm{~h}_{1}(x)}{\partial x_{12}(x)} & \frac{\partial L_{f} \mathrm{~h}_{1}(x)}{\partial x_{2}(x)} & \frac{\partial L_{f} \mathrm{~h}_{1}(x)}{\partial x_{22}(x)} & \frac{\partial L_{f} \mathrm{~h}_{1}(x)}{\partial x_{1} 1} & \frac{\partial L_{f} \mathrm{~h}_{1}(x)}{\partial x_{32}(x)} \\
\frac{\partial L_{f} \mathrm{~h}_{2}(x)}{\partial x_{11}} & \frac{\partial L_{f} \mathrm{~h}_{2}(x)}{\partial x_{12}} & \frac{\partial L_{f} \mathrm{~h}_{2}(x)}{\partial x_{21}} & \frac{\partial L_{f} \mathrm{~h}_{2}(x)}{\partial x_{22}} & \frac{\partial L_{f} \mathrm{~h}_{2}(x)}{\partial x_{1}} & \frac{\partial L_{f} \mathrm{~h}_{2}(x)}{\partial x_{32}} \\
\frac{\partial L_{f}^{2} h_{1}(x)}{\partial x_{11}} & \frac{\partial L_{f}^{2} h_{1}(x)}{\partial x_{12}} & \frac{\partial L_{f}^{2} h_{1}(x)}{\partial x_{2}} & \frac{\partial L_{f}^{2} h_{1}(x)}{\partial x_{22}} & \frac{\partial L_{f}^{2} h_{1}(x)}{\partial x_{3}} & \frac{\partial L_{f}^{2} h_{1}(x)}{\partial L_{32}} \\
\frac{\partial L_{f}^{2} h_{2}(x)}{\partial x_{11}} & \frac{\partial L_{f}^{2} h_{2}(x)}{\partial x_{12}} & \frac{\partial L_{f}^{2} h_{2}(x)}{\partial x_{21}} & \frac{\partial L_{f}^{2} h_{2}(x)}{\partial x_{22}} & \frac{\partial L_{f}^{2} h_{2}(x)}{\partial x_{31}} & \frac{\partial L_{f}^{2} h_{2}(x)}{\partial x_{32}}
\end{array}\right]
\end{aligned}
$$

It is obvious that the Jacobian matrix, $J_{O_{W T G}} h(x)$ is of full rank if and only if the square matrix is also full rank nonsingular,

$$
\begin{aligned}
& J_{O_{W T G}} h(x)^{\Delta} \\
& =\left[\begin{array}{llll}
\frac{\partial L_{f} \mathrm{~h}_{2}(x)}{\partial x_{21}} & \frac{\partial L_{f} \mathrm{~h}_{2}(x)}{\partial x_{22}} & \frac{\partial L_{f} \mathrm{~h}_{2}(x)}{\partial x_{31}} & \frac{\partial L_{f} \mathrm{~h}_{2}(x)}{\partial x_{32}} \\
\frac{\partial L_{f}^{2} h_{1}(x)}{\partial x_{21}} & \frac{\partial L_{f}^{2} h_{1(x)}}{\partial x_{22}} & \frac{\partial L_{f}^{2} h_{1}(x)}{\partial x_{31}} & \frac{\partial L_{f}^{2} h_{1(x)}}{\partial x_{32}} \\
\frac{\partial L_{f}^{2} h_{2}(x)}{\partial x_{21}} & \frac{\partial L_{f}^{2} h_{2}(x)}{\partial x_{22}} & \frac{\partial L_{f}^{2} h_{2}(x)}{\partial x_{31}} & \frac{\partial L_{f}^{2} h_{2}(x)}{\partial x_{32}}
\end{array}\right]
\end{aligned}
$$

Now, it will be proved that, $J_{O_{W T G}} h(x)$ is regular square matrix, which can be computed the determinant of $J_{O_{W T G}} h(x)$ after simple algebraic manipulation, yields:

$\operatorname{det}\left\{J_{O_{W T G}} h(x)\right\}=\frac{n_{p}^{4} R_{a}}{J L_{a}^{5}}\left(x_{21}\right)^{2}\left(x_{32-} x_{31}\right)$

It can be explained by using the original PMSG system state variables as follows:

$\operatorname{det}\left\{J_{W T G} h(x)\right\}=\frac{n_{p}^{4} R_{a}}{J L_{a}^{5}}\left(\omega_{g}\right)^{2} \psi_{r}$

Notice that, one can concentrate on the matrix $J_{O_{W T G}} h(x)$ in order to introduce a sufficient condition such that this matrix, or equivalently, $J_{O_{W T G}} h(x)$, is of full rank almost everywhere. Actually, this means that observability is independent of the system inputs and thus can permit design of uniform observer also independent of the systems inputs.

Remark 2 From this, one can say that if, $\operatorname{det}\left(J_{O_{W T G}} h(x)\right) \neq$ 0 , wind turbine generator system WTG, is observable in the rank sense. It has been emphasized that, as the norm of the rotor flux is constant and never vanish at all times. Practically, the observability concept is lost only at the singular point corresponding to zero speed and the $\operatorname{det}\left(J_{O_{W T G}} h(x)\right) \neq 0$ will never vanish if and only if the generator speed is null that implies in standstill.

\subsection{Model transformation of PMSG dynamics}

A model transformation is required to construct an observer normal form; however it is proved that for some classes of nonlinear systems, it is necessary to support the state transformation. The present system is expressed in the observer normal form; a sampled high gain observer can be designed. It consists of a copy of the dynamics of the original system corrected by an output injection term with the matrix highgain of the form $\theta \Delta^{-1} K$.

Once, a state transformation is required to construct an observer normal form; however it is proved that for some classes of nonlinear systems, it is necessary to support the state transformation. This system is expressed in the observability normal form, a sampled-data high gain observer can be designed. It consists of a copy of the dynamics of the original system corrected by an output injection term. Let us define the following state transformation of the observability map:- 
$\Phi: \mathbf{R}^{6} \rightarrow \mathbf{R}^{6},\left(\begin{array}{c}x \\ u_{g}\end{array}\right) \mapsto\left(\begin{array}{c}z \\ u_{g}\end{array}\right)$

$$
\triangleq\left(\begin{array}{l}
z_{11} \\
z_{12} \\
z_{21} \\
z_{22} \\
z_{31} \\
z_{32}
\end{array}\right)=\Phi\left(\mathrm{x}, u_{g}\right)
$$

$\Phi\left(\mathrm{x}, u_{g}\right) \triangleq\left[h_{1}(x) h_{2}(x) L_{f} h_{1}(x) L_{f} h_{2}(x) L_{f}^{2} h_{1}(x) L_{f}^{2} h_{2}(x)\right]^{T}$

One can demonstrate that the above state transformation that puts system (5236) under the following form one gets:

$\dot{z}_{1}=\left[L_{f} h_{1}(x), L_{f} h_{2}(x)\right]^{T}=z_{2}+\varphi_{1}\left(z_{1}, u_{g}\right)$

$\dot{z}_{2}=\left[L_{f}^{2} h_{1}(x), L_{f}^{2} h_{2}(x)\right]^{T}=z_{3}+\varphi_{2}\left(z_{1}, z_{2}\right)$

$\dot{z}_{3}=\varphi_{3}(z)+B_{c} b(z) T_{g o p t}$

Knowing that the state transformation mapping elements are,

$$
\left\{\begin{array}{l}
\Phi_{1}(x)=h(x) \triangleq\left[L_{f}^{0} h_{1}(x), L_{f}^{0} h_{2}(x)\right]^{T} \\
=\left[h_{1}(x), h_{2}(x)\right]^{T} \\
\Phi_{2}(x)=\left[L_{f} h_{1}(x), L_{f} h_{2}(x)\right]^{T} \\
\Phi_{3}(x)=\left[L_{f}^{2} h_{1}(x), L_{f}^{2} h_{2}(x)\right]^{T}
\end{array}\right.
$$

After this state transformation and for a suitable situation, the system model (64) will present in more compact form:

$\Sigma_{z}\left\{\begin{array}{l}\dot{z}=A_{c} z+\varphi\left(z, u_{g}\right)+B_{c} b(z) T_{g o p t} \\ y=C_{c} z=z_{1}\end{array}\right.$

where the whole system state variable vector, $z \triangleq$ $\left[\begin{array}{llllll}z_{11} & z_{12} & z_{21} & z_{22} & z_{31} & z_{32}\end{array}\right]^{T} \in \boldsymbol{R}^{6}$, the matrices $A_{c}, B_{c}$ appearing in the previous state-space equation of system (52) and the function $\varphi\left(z, u_{g}\right) \in R^{6}$ has block triangular structure with respect to $\mathrm{z}$ uniformly in input $u_{g}$.

$$
A_{c}=\left[\begin{array}{llllll}
0 & 1 & 0 & 0 & 0 & 0 \\
0 & 0 & 1 & 0 & 0 & 0 \\
0 & 0 & 0 & 1 & 0 & 0 \\
0 & 0 & 0 & 0 & 1 & 0 \\
0 & 0 & 0 & 0 & 0 & 1 \\
0 & 0 & 0 & 0 & 0 & 0
\end{array}\right] \in \boldsymbol{R}^{6}
$$

The state sampled HGO nonlinear observer for the transformed system of (65) is

$$
\left\{\begin{array}{l}
\dot{\hat{z}}=A_{c} \hat{z}+\varphi\left(\operatorname{sat}(\hat{z}), u_{g}\right)-\theta \Delta^{-1} K(h(\hat{z})-w) \\
\dot{w}=L_{f} h(\Phi(\hat{x}))=C_{c}\left(A_{c} \hat{z}+\varphi_{o}\left(\operatorname{sat}(\hat{z}), u_{g}\right)\right. \\
\forall t \in\left[t_{k}, t_{k+1}\right), \mathrm{k} \in \mathbb{N} \\
\quad w\left(t_{k+1}\right)=y\left(t_{k+1}\right) \\
\dot{\theta}=g(\theta), \theta\left(t_{0}\right)>1 \forall t \in R^{+} \\
\hat{y}=C_{c} \hat{z}=\hat{z}_{1}=\left(\hat{\iota}_{g \alpha}, \hat{\iota}_{g \beta}\right)^{T} \\
\hat{z}\left(t_{0}\right)=\hat{z}_{0}
\end{array}\right.
$$

It is also possible to express the state SDHGO using a nonlinear model, i.e.,

$\dot{\hat{x}}=f(\hat{x})-L \theta \Delta^{-1}(h(\hat{x})-w)$

It will be shown that the nonlinear observer gain $L$ is given by:

$L=\left(J_{N_{O_{W T G}}} h(\hat{x})\right)^{-1} K$

where the matrix $J_{W T G} h(\hat{x})$ is the Jacobian of the new coordinates, $z_{11}, z_{12}, z_{21}, z_{22}, z_{31}, z_{32}$, which is obtained after the nonlinear state transformation and $\theta \Delta^{-1} K$ is the HGO evaluated for the linearized equivalent of the system (67). The matrix high-gain $\theta \Delta^{-1} K$ can be obtained through the following procedure:

$$
\begin{aligned}
d \hat{z}= & {\left[d h_{1}(\hat{x}) d h_{2}(\hat{x}) d L_{f} h_{1}(\hat{x}) d L_{f} h_{2}(\hat{x}) d L_{f}^{2} h_{1}(\hat{x})\right.} \\
& \left.d L_{f}^{2} h_{2}(\hat{x})\right]^{T}
\end{aligned}
$$

Or, equivalently,

$\dot{\hat{z}}=J_{N_{O_{W T G}}} h(\hat{x}) \dot{\hat{x}}$

It holds that the Jacobian matrix of, $\frac{\partial \Phi(\hat{x})}{\partial \hat{x}}=\nabla O_{W T G} h(\hat{x})$ Using the state observer dynamics described in (68), one gets:

$\frac{\partial \Phi(\hat{x})}{\partial \hat{x}} \dot{\hat{x}}=\frac{\partial \Phi(\hat{x})}{\partial \hat{x}} f(\hat{x})+\frac{\partial \Phi(\hat{x})}{\partial \hat{x}} L(\hat{x})(h(\hat{x})-w)$

Let us consider that for the first row of the Jacobian matrix, it holds, $\Phi(\hat{x})=h(\hat{x})$, one obtains:

$\frac{\partial h(\hat{x})}{\partial \hat{x}} \dot{\hat{x}}=\frac{\partial h(\hat{x})}{\partial \hat{x}} f(\hat{x})-\theta \Delta^{-1} K(h(\hat{x})-w)$

Or, equivalently,

$\dot{\hat{z}}_{1}=L_{f} h(\hat{x})+\varphi_{1}\left(z_{1}, u_{g}\right)-\theta \Delta^{-1} K_{1}(h(\hat{x})-w)$

Furthermore, it holds $L_{f} h(\hat{x})=\hat{z}_{2}, \Delta^{-1}=\mathrm{I}_{2}$, this implies that: 
$\dot{\hat{z}}_{1}=\hat{z}_{2}+\varphi_{1}\left(z_{1}, u_{g}\right)-\theta K_{1}(h(\hat{x})-w)$

$\dot{\hat{z}}_{2}=\hat{z}_{3}+\varphi_{1}\left(z_{1}, z_{2}\right)-\theta^{2} K_{2}(h(\hat{x})-w)$

$\dot{\hat{z}}_{3}=\varphi_{3}(z)-\theta^{3} K_{3}(h(\hat{x})-w)$

Using the previous notation, one gets the mathematical formulation of the nonlinear observation's gain $L$ as a function of the observation gain $K$ for the linearized equivalent of the system observer of (68), so finally one has the same equation specified in (82).

$$
\begin{aligned}
\left(N_{O_{W T G}} h(\hat{x})\right) L \theta \Delta^{-1} & =\theta \Delta^{-1} K \rightarrow \\
L & =\left(N_{O_{W T G}} h(\hat{x})\right)^{-1} K
\end{aligned}
$$

The next step will propose a nonlinear sampled-data high gain observer design based on sensorless output prediction.

\subsection{Semi-global sampled high gain observer synthesis}

The main purpose of this section is to provide a SDHGO for the system model given in (52). This observer will lead to online estimation of generator state variables, which are $i_{g}, \psi_{r}, \omega_{\mathrm{g}}$ and $T_{g}$ including the rotor position using the generator input output measurements of the generator stator currents $i_{g \alpha} \triangleq\left(i_{g \alpha}, i_{g \beta}\right)^{T}$ and the generator input control signal $u_{g} \triangleq\left[\begin{array}{ll}u_{g \alpha} & u_{g \beta}\end{array}\right]^{T}$.

Indeed, because of the state feedback controller is generally designed using the $(d-q)$ rotor reference frame, for its simplicity in controller design to estimate online several state variables involving the generator rotor position. If the estimates of the generator rotor flux $\left(\hat{\psi}_{r \alpha}, \hat{\psi}_{r \beta}\right)^{\mathrm{T}}$ become available, the mechanical rotor position calculated using [31]. The position angle is bounded by the estimates of the rotor flux vector $\left[\hat{\psi}_{\mathrm{r} \alpha}, \hat{\psi}_{\mathrm{r} \beta}\right]^{T}$ in stationary reference frame, the mechanical rotor position can be obtained using following well-known formula; where $n_{p}$ is number of pole pairs. The mechanical rotor position of the PMSG is the mechanical rotor position of the PMSG as:

$\hat{\theta}_{r m}=\frac{1}{n_{p}} \hat{\theta}_{r e}=\frac{1}{n_{p}} \tan ^{-1}\left(\frac{\hat{\psi}_{\mathrm{r} \beta}}{\hat{\psi}_{\mathrm{r} \alpha}}\right)$

For a suitable situation, the system model (56) will present in compact form for MIMO system is (after doing model transformation):

$\Sigma_{z}\left\{\begin{array}{l}\dot{z}=A_{c} z+\varphi\left(z, u_{g}\right)+B_{c} b(z) T_{\text {gopt }} \\ y=C_{c} z=z_{n}\end{array}\right.$

This study defines the following mathematical notations which are widely used for high gain observer design:
Let us consider $\Delta$ be the block diagonal matrix defined:

$\Delta \triangleq \operatorname{diag}\left(I_{2}, \frac{1}{\theta} I_{2}, \frac{1}{\theta^{2}} I_{2}\right) \in R^{6}$

with, $\theta\left(t_{0}\right)>1, \forall t_{0} \geq 0$ is DHGO parameter.

Let us take $\mathrm{K} \in R^{6 \times 2}$ be gain matrix such that $\left(A_{c}-K C_{c}\right)$ is Hurwitz (all its eigenvalues have negative real parts). This choice is always possible since the pair $\left(A_{c} ; C_{c}\right)$ is observable. The gain matrix $\mathrm{K}$ can be chosen as:

$K^{T} \triangleq\left[\begin{array}{cccccc}k_{11} & 0 & k_{21} & 0 & k_{31} & 0 \\ 0 & k_{12}, & 0 & k_{22} & 0 & k_{32}\end{array}\right]$

where, $k_{i j}(i=1 ; 2 ; 3$ and $j=1 ; 2)$ is a real positive constant corresponding to gain of measurement error. For each $K$, there exists a symmetric positive definite matrix $P \in R^{6 \times 6}, P=P^{T}>0, \mu>0$ positive free constant, $I_{6} \in R^{6 \times 6}$ is identity matrix such that the following algebraic Lyapunov equation is satisfied:

$P\left(A_{c}-K C_{c}\right)+\left(A_{c}-K C_{c}\right)^{T} P \leq-\mu I_{6}$

where $A_{c}$ and $C_{c}$ are respectively, defined above. This is useful tool for checking convergence properties and for checking Lyapunov stability test.

One supposes that the following inductive hypotheses are valid, which are usually used when designing high-gain state observers. Using these hypotheses, the searcher will present some new results on designing state observers for a class of Lipschitz, nonlinear systems.

$\boldsymbol{H}_{\mathbf{1}}$ : The functions $\varphi_{i}\left(z, u_{g}\right): R^{6} \times R^{6} \rightarrow R^{6}$ are locally Lipschitz and globally bounded with respect to $z$ in domain of interest, uniformly in $u_{g}$ i.e., $\exists \beta_{0}>0$, Lipchitz positive constant is a maximum of $\left\|\frac{\partial f}{\partial z}\left(x, u_{g}\right)\right\|$, such that, $\forall(z, \hat{z}) \in$ $R^{n} \times R^{n}, \forall u_{g} \in U, q \in R$, one can easily write the following nonlinear matrix inequality for $\theta\left(t_{0}\right)>1$, which is useful for synthesis semi-global observer based on Lipschitz extension function as:

$\left\|\frac{\Delta}{\theta^{r}}\left[\varphi_{o}\left(\operatorname{sat}(\hat{z}), u_{g}\right)-\varphi\left(\operatorname{sat}(z), u_{g}\right)\right]\right\| \leq \sqrt{q} \beta_{0}\left\|\epsilon_{z}\right\|$

Or, $\left\|\left[\varphi_{o}\left(\operatorname{sat}(\hat{z}), u_{g}\right)-\varphi\left(\operatorname{sat}(z), u_{g}\right)\right]\right\| \leq \sqrt{q} \beta_{0}\left\|e_{z}\right\|$

where $\operatorname{sat}(z)$ is an element wise saturation function, which is saturated outside of $z$. Note that the dynamics $\dot{z_{n}}$ depends on all saturated state variables $\operatorname{sat}\left(z_{1}\right), \ldots, \operatorname{sat}\left(z_{6}\right)$ through Lipchitz extension function, $\varphi\left(\operatorname{sat}(z), u_{g}\right)$. The motivations of using $\boldsymbol{H}_{\mathbf{1}}$ are first one is related to the observability analysis of sensorless PMSG and second one is that proposed sampled HGO needs the Lipschitz property of the vector matrix 
$f\left(x, u_{g}\right) \rightarrow f\left(\operatorname{sat}(x), u_{g}\right)$, because the observer gain chosen is based on the dynamic HGO approach.

For physical point of view and domain of working principle, it is supposed that all physical state variables are bounded in domain of interest as stated in $\boldsymbol{H}_{2}$. To overcome the blow up state variables in finite time that may reduce the escape time of the system and to restrict the initial peaking phenomenon. They are practically reasonable.

$\boldsymbol{H}_{\mathbf{2}}$ : The functions $\varphi_{i}\left(\operatorname{sat}(z), u_{g}\right)$ are lowering triangular structure in $z$, i.e, $\frac{\partial \varphi_{i}\left(\operatorname{sat}(z), u_{g}\right)}{\partial z_{k+1}}=0, k=i, \ldots, n-1$ to provide semi-global observer design

$\varphi\left(\operatorname{sat}(z), u_{g}\right)=\left(\begin{array}{c}\varphi_{1}\left(\operatorname{sat}\left(z_{1}\right), u_{g}\right) \\ \varphi_{2}\left(\operatorname{sat}\left(z_{1}\right), \operatorname{sat}\left(z_{2}\right), u_{g}\right) \\ \vdots \\ \varphi_{n}\left(\operatorname{sat}(z), u_{g}\right)\end{array}\right) \in R^{6}$

Using the saturation function, sat (.), one limits the maleffect of the wrong state estimate, and reduce the effect of peaking phenomenon at the starting time of operation since the large observation and tracking errors in previous state will make the observation errors in current state difficult to estimate. In fact, this hypothesis is highly recommended for stability convergence analysis.

\subsection{Sampled-output high-gain observer structure}

The purposed structure is to design a semi-global sampled high-gain observer having dynamic continuous-time design parameter $\theta$ for the system given in (52). The semi-global exponential convergence takes place whenever $\theta$ is satisfactory large enough value. Thus $\theta$ must evolve between greater than unity and high enough value, whose existence has to be proven below.

Let us consider the following semi-global sampled highgain observer SDHGO having dynamic continuous-time high gain design parameter and coupled with predictor introduced by [32] and the references therein for more details:

$$
\left\{\begin{array}{l}
\dot{\hat{z}}=A_{c} \hat{z}+\varphi_{o}\left(\operatorname{sat}(\hat{z}), u_{g}\right)-\theta \Delta^{-1} K\left(C_{c}(\hat{z})-w\right) \\
\dot{w}=C_{c}\left(A_{c} \hat{z}+\varphi_{o}\left(\operatorname{sat}(\hat{z}), u_{g}\right) \forall t \in\left[t_{k}, t_{k+1}\right), \mathrm{k} \in \mathbb{N}\right. \\
w\left(t_{k+1}\right)=y\left(t_{k+1}\right) \\
\dot{\theta}=g(\theta), \theta\left(t_{0}\right)>1 \forall t \in R^{+} \\
\hat{y}=C_{c} \hat{z}=\hat{z}_{1}=\left(\hat{\imath}_{g \alpha}, \hat{\imath}_{g \beta}\right)^{T} \\
\hat{z}\left(t_{0}\right)=\hat{z}_{0}
\end{array}\right.
$$

with the state vector, $\hat{z} \in R^{6}$ is the continuous-time estimate of the real system state, $\mathrm{z} \in R^{6}$ and $\Delta, K$ are well-defined in (79), (80), respectively. The vector $w=\left[w_{1}, w_{2}\right]^{T}$, represents the output prediction between two consecutive sampling points. The output state prediction $w$ is updated at each sampling instant $t_{k}$ to predict future output signal.
The functiong: $R \rightarrow R$ is sufficiently smooth function i.e., (infinite continuous time derivative function with initial value must greater than unity). Indeed, the dynamical continuous-time design parameter is increasing in the time interval $\left[t_{k}, t_{k+1}\right), \quad \mathbb{N}$ is set of nonnegative integers such that $\theta\left(t_{0}\right)>1, t_{0} \geq 0, \forall t \in R^{+}$and $t_{k+1}=t_{k}+T_{s}$.

It should be confirmed that the authors in [24] and [26] focused on state-affine system model running with interconnected Kalman-like observer injected by persistence excitation inputs and system output state measurements in continuous-time mode. Actually, the searchers considered the output state vector is accessible without taking into account the concept of inter-sampled behaviour in design process for such state observer synthesis.

Theorem (Main Result): Given class of nonlinear system stated in (76) with compact form, which submits to inductive hypotheses named $\boldsymbol{H}_{1}, \boldsymbol{H}_{2}, \boldsymbol{H}_{3}$ such that the evaluation of dynamical continuous time design parameter has been chosen as closed-loop gain smooth function:

$\dot{\theta} \triangleq-\frac{\theta}{r}\left[\frac{\mu}{2} \theta+2 r \frac{\dot{\theta}+|\dot{\theta}| \beta_{0} \lambda \max (P)}{\theta \lambda \min (P)}\right]$

The maximal admissible sampling period $T_{\text {max }}$ satisfying the following nonlinear inequality in terms of other sampled HGO parameters to ensure semi-global exponential convergence of the observation error for $q \in R, \beta_{0}>0, r>0$ are strictly positive real number chosen sufficiently large enough.

$T_{\max } \leq \frac{\sqrt{\mu \lambda \min (P)}}{\sqrt{2 \theta_{\max }} \lambda \max (P)\|K\|\left(\theta_{\max }+\sqrt{6} \beta_{0}\right)}$

thus, $\forall \theta_{\max } \geq \theta\left(t_{0}\right)>1$, and $\forall T_{s} \in\left(0, T_{\text {max }}\right]$ is piecewise function, the system stated in (84) is semi-global exponential observer (SGEO) for the system model defined in (78). Dynamic HGO for continuous-time systems is designed in order to address the issue of the sensitivity measurements error inherent this kind of nonlinear state observers.

Proof This study will give a formal analysis and elegant full prove of the main theorem using tools of Lyapunov stability nonlinear control approach. Let us consider the system states observations error between estimated and actual state variables are:

$e_{z}(t) \triangleq\left[e_{z 1}(t) e_{z 2}(t) e_{z 3}(t)\right]^{T} \triangleq \hat{z}(t)-z(t)$

The time derivative of the observation error is given by:

$\dot{e}_{z}(t)=\dot{\hat{z}}(t)-\dot{z}(t)$ 
For writing convenience, the time index, $t$ can be cancelled and let us define, $e_{w}$ denotes the output prediction error between the output state predictor and the actual output measurements:

$e_{w} \triangleq w-y \triangleq w-C_{c} z=\left[\begin{array}{l}w_{\alpha}-z_{11} \\ w_{\beta}-z_{12}\end{array}\right]$

In view of systems (78) and (84), the time derivative of output prediction error gives: $\dot{e}_{w}=\dot{w}-\dot{y}=\dot{w}-C_{c} \dot{z}$

Notice that from (89), one defines: $w \stackrel{\text { def }}{=} e_{w}+y=e_{w}+$ $C_{c} z$ used in the new error dynamics structure shown below $\forall t \in\left[t_{k}, t_{k+1}\right), \mathrm{k} \in \mathbb{N}, \forall t \in R^{+}$:

$$
\begin{aligned}
\dot{e}_{z}= & \left(A_{c}-\theta \Delta^{-1} K C_{c}\right) e_{z}+\varphi_{o}\left(\operatorname{sat}(\hat{z}), u_{g}\right) \\
& -\varphi\left(\operatorname{sat}(z), u_{g}\right) \\
& +\theta \Delta^{-1} K e_{w}-B_{c} b(z) T_{g o p t} \\
\dot{e}_{w}= & C_{c}\left(A_{c} e_{z}+C_{c}\left\{\varphi_{o}\left(\operatorname{sat}(\hat{z}), u_{g}\right)\right.\right. \\
& \left.-\varphi\left(\operatorname{sat}(z), u_{g}\right)\right\}-C B_{c} b(z) T_{g o p t} \\
\dot{\theta}= & g(\theta), \theta\left(t_{0}\right)>1
\end{aligned}
$$

It is simply checked the following mathematical identities: $\Delta^{-1} A_{c} \Delta=\theta^{-1} A_{c}$ and $C_{c} \Delta=C_{c} \Delta^{-1}=C_{c}$, therefore, it is easily checked that the new system error dynamics can be re-written $\forall \mathrm{t} \in\left[t_{k}, t_{k+1}\right), \mathrm{k} \in \mathbb{N}$ as follows:

$$
\begin{aligned}
\dot{e}_{z}= & \theta \Delta^{-1}\left(A_{c}-K C_{c}\right) \Delta e_{z}+\varphi_{o}\left(\operatorname{sat}(\hat{z}), u_{g}\right) \\
& -\varphi\left(\operatorname{at}(z), u_{g}\right) \\
& +\theta \Delta^{-1} K e_{w}-B_{c} b(z) T_{g o p t} \\
\dot{e}_{w}= & e_{z 2}+\varphi_{o 1}\left(\operatorname{sat}(\hat{z}), u_{g}\right)-\varphi_{1}\left(\operatorname{at}(z), u_{g}\right) \\
& -C B_{c} b(z) T_{g o p t} \dot{\theta}=g(\theta), \theta\left(t_{0}\right)>1, \quad \forall t \in R^{+}
\end{aligned}
$$

Let us apply the following principle of change of coordinates:

$\epsilon_{z} \triangleq \frac{1}{\theta^{r}} \Delta e_{z}$

In order to facilitate the mathematical calculations, as $\Delta$ is invertible block diagonal matrix and the number $r$ is a strictly positive real number chosen sufficiently large enough value, that will be defined later in this paper:

Notice that:

$\dot{\epsilon}_{z}=\frac{1}{\theta^{r}} \Delta \dot{e}_{z}+\frac{d}{d t}\left(\frac{1}{\theta^{r}} \Delta\right) e_{z}$

So, in view of the first subsystem of (52), the first term of the right hand side of (54) gives:

$$
\begin{aligned}
\frac{1}{\theta^{r}} \Delta \dot{e}_{z}= & \theta\left(A_{c}-K C_{c}\right) \epsilon_{z} \\
& +\frac{1}{\theta^{r}} \Delta\left\{\varphi_{o}\left(\operatorname{sat}(\hat{z}), u_{g}\right)-\varphi\left(\operatorname{at}(z), u_{g}\right)\right\} \\
& +\frac{1}{\theta^{r-1}} K e_{w}-\frac{1}{\theta^{r}} \Delta B_{c} b(z) T_{g o p t}
\end{aligned}
$$

On other hand, the time derivative of the second term in the right hand side of system (93) combined with the definition of block diagonal matrix stated in (79) for $\Delta$ will give:

$$
\begin{aligned}
\frac{d}{d t}\left(\frac{1}{\theta^{r}} \Delta\right) e_{z} & =\frac{d}{d t}\left(\frac{1}{\theta^{r}} \operatorname{diag}\left\{\mathrm{I}_{2}, \frac{1}{\theta} \mathrm{I}_{2}, \frac{1}{\theta^{2}} \mathrm{I}_{2}\right\}\right) e_{z} \\
& =\frac{d}{d t}\left(\operatorname{diag}\left\{\theta^{-r}, \theta^{-r-1}, \theta^{-r-2}\right\} \mathrm{I}_{2}\right) e_{z}
\end{aligned}
$$

Therefore,

$\frac{\mathrm{d}}{\mathrm{dt}}\left(\frac{1}{\theta^{\mathrm{r}}} \Delta\right) \mathrm{e}_{\mathrm{z}}=\frac{-\dot{\theta}}{\theta^{\mathrm{r}+1}} \operatorname{diag}\{\mathrm{r}, \mathrm{r}+1, \mathrm{r}+2\} \Delta \mathrm{e}_{\mathrm{z}}$

from (95) and (92), one concludes that:

$\frac{d}{d t}\left(\frac{1}{\theta^{r}} \Delta\right) e_{z}=\frac{-\dot{\theta}}{\theta} D\left(\frac{1}{\theta^{r}} \Delta e_{z}\right)=\frac{-\dot{\theta}}{\theta} D \epsilon_{z}$

where, $\mathrm{D} \triangleq \operatorname{diag}\{r, r+1, r+2, \ldots, n-1\} . D \triangleq$ $r I_{n}+E$, and

$E \triangleq \operatorname{diag}\{0,1,2, \ldots, n-1\}$

Combining (94) and (96), one has the solution of system (93):

$$
\begin{aligned}
\dot{\epsilon}_{z}= & \theta\left(A_{c}-K C_{c}\right) \epsilon_{z} \\
& +\frac{1}{\theta^{r}} \Delta\left(\varphi_{o}\left(\operatorname{sat}(\hat{z}), u_{g}\right)-\varphi\left(\operatorname{sat}(z), u_{g}\right)\right) \\
& +\frac{1}{\theta^{r}} \theta K e_{w}-\frac{1}{\theta^{r}} \Delta B_{c} b(z) T_{g o p t}-\frac{\dot{\theta}}{\theta} D \epsilon_{z}
\end{aligned}
$$

So, $\forall t \in\left[t_{k}, t_{k+1}\right), \mathrm{k} \in \mathbb{N}$, the new system dynamics error is:

$$
\begin{aligned}
\dot{\epsilon}_{z}= & \theta \Delta^{-1}\left(A_{c}-K C_{c}\right) \Delta e_{z}+\varphi_{o}\left(\operatorname{sat}(\hat{z}), u_{g}\right) \\
& -\varphi\left(\operatorname{sat}(z), u_{g}\right)+C_{e} \\
\dot{e}_{w}= & e_{z 2}+\varphi_{o 1}\left(\operatorname{sat}(\hat{z}), u_{g}\right) \\
& -\varphi_{1}\left(z, u_{g}\right)-C B_{c} b(z) T_{g o p t} \\
\dot{\theta}= & g(\theta), \theta\left(t_{0}\right)>1 \forall t \in R^{+}
\end{aligned}
$$

with, $C_{e}=\theta \Delta^{-1} K e_{w}-B_{c} b(z) T_{g o p t}-\frac{\dot{\theta}}{\theta} D \epsilon_{z}$ is the innovation correction term for the proposed sampled HGO observer. 


\subsection{Design of semi-global output feedback controller}

Following the output feedback control architecture, the un-accessible to measurements mechanical states are now replaced by their online estimates provided by the SDHGO observer (84). Doing so, the output feedback controller turns out to be defined by the control laws based on control laws of (21) and (30) of the system (2). The semi-global output feedback stabilization problem, and used a saturation function in the bounded practical control law in order to eliminate and limit the mal-effects due to the wrong estimates of the highgain observer in previous blocks. One constructs a desired semi-global output feedback controller using saturated state estimate [33]:

$$
\begin{aligned}
& \hat{u}_{g q} \triangleq u_{1} \mathrm{~V}_{\mathrm{dc}}=\frac{J}{n_{p} \psi_{\mathrm{PM}}}\left[-\left(h_{1}+h_{2}\right) \text { sat }\left(i_{q r e f}-\hat{\iota}_{q}\right)\right. \\
& +\left(h_{1}^{2}-1\right) \operatorname{sat}\left(\hat{\omega}_{g}-\omega_{\text {opt }}\right) \\
& \left.-\xi\left(\operatorname{sat}\left(\hat{\iota}_{d}\right), \operatorname{sat}\left(\hat{\iota}_{q}\right), \operatorname{sat}\left(\hat{\omega}_{g}\right)\right)\right] \\
& \hat{u}_{g d} \triangleq u_{2} V_{d c}=\mathrm{L}_{\mathrm{a}}\left[\mathrm{p} \operatorname{sat}\left(\hat{\iota}_{q}\right), \operatorname{sat}\left(\hat{\omega}_{g}\right)\right. \\
& \left.-\frac{1}{K_{t}} \operatorname{sat}\left(\hat{\iota}_{d}-i_{d r e f}\right)+h_{3} \operatorname{sat}\left(i_{q r e f}-\hat{\iota}_{q}\right)\right] \\
& \hat{u}_{n 1} \triangleq u_{3} \mathrm{~V}_{\mathrm{dc}}=\mathrm{V}_{\mathrm{dc}}\left\{h_{5} \operatorname{sat}\left(\rho_{2}-\rho_{2}^{r e f}\right)+V_{d c}^{2}-V_{d c r e f}^{2}\right. \\
& \left.+\Upsilon_{1}\left(\operatorname{sat}\left(\hat{\imath}_{d}, \hat{\iota}_{q}, \hat{\omega}_{g}, V_{d c}^{2}, i_{n d}, i_{n q}, \hat{\varepsilon}_{\omega}, \hat{\varepsilon}_{q}, \hat{\varepsilon}_{d}, \varepsilon_{V d c}, \varepsilon_{n d}\right)\right)\right\} \\
& \frac{2 C_{b u s} L_{i n v}}{E_{n d} V_{d c}} \\
& \hat{u}_{n 2} \triangleq u_{4} V_{d c} \\
& \quad=\mathrm{V}_{\mathrm{dc}} \frac{-L_{i n v}\left(h_{6} s a t\left(Q_{n}-Q_{n r e f}\right)+\Upsilon_{2}\left(\operatorname{sat}\left(i_{n d}, i_{n q}\right)\right)\right.}{E_{n d}}
\end{aligned}
$$

The saturation actuator function sat (.) : $\mathrm{R} \rightarrow[-\rho, \rho]$ is defined:

$\operatorname{Sat}\left(\frac{s}{M_{a}}\right) \triangleq\left\{\begin{array}{l}\rho, \quad \text { if } s>M_{a} \\ s, \quad \text { if }|s| \leq M_{a} \\ -\rho, \quad \text { if } s<-M_{a}\end{array}\right.$

where, $\rho$ is upper bound. It is used in many practical cases. Unluckily, the nonlinearity appearing in system (12) is locally Lipschitz on the whole $R^{6}$ according to $\boldsymbol{H}_{\mathbf{1}}$ and to avoid this situation, one shall apply the Lipschitz prolongation technique [34]. Doing so, one assumes that the inputs control laws $\hat{u}_{g d}$ and $\hat{u}_{g q}$ are bounded and let $U_{g d}>0$ and $U_{g q}>0$, be the corresponding upper bound, that is:

$$
U_{g d} \triangleq \sup _{t \geq 0,}\left\|u_{g d}(t)\right\| \text { and } U_{g q} \triangleq \sup _{t \geq 0,}\left\|u_{g q}(t)\right\|
$$

Finally, let us consider the dynamic output feedback control laws in $(d-q)$ coordinates proposed here as:

$$
\begin{aligned}
& u_{O F C} \triangleq\left[\hat{u}_{g d}, \hat{u}_{g q}\right]^{T}=\zeta\left(\hat{T}_{g}, \hat{\omega}_{g}, \hat{\iota}_{q}, \hat{\iota}_{d}\right) \\
& \hat{u}_{g d}=U_{g d} \frac{\operatorname{sat}\left(\zeta\left(\hat{T}_{g}, \hat{\omega}_{g}, \hat{\iota}_{q}, \hat{\iota}_{d}\right)\right)}{U_{g d}} \text { and } \\
& \hat{u}_{g q}=U_{g q} \frac{\operatorname{sat}\left(\zeta\left(\hat{T}_{g}, \hat{\omega}_{g}, \hat{\iota}_{q}, \hat{\iota}_{d}\right)\right)}{U_{g q}}
\end{aligned}
$$

A global sampled HGO observer was presented with the global Lipschitz assumption of the system model. Nevertheless, this assumption will be eliminated through the Lipschitz extension technique if the semi-global sampled HGO observer is considered. It is able to restrict the class of systems to which the proposed approach becomes appropriate. This can be accomplished if one proposes semi-global observer instead of global observer and the system model is adjusted outside the region of attraction. This adjustment is called Lipschitz extension technique, which had been introduced by [35]. Suppose that a semi-global sampled high-gain observer is synthesized which estimates the system states, as

$$
\begin{aligned}
\hat{u}_{g d}(t) & \rightarrow u_{g d}(t) \text { And } \hat{u}_{g q}(t) \rightarrow u_{g q}(t) \\
\text { and, } \dot{z} & :=A_{c} \hat{z}+\varphi\left(\hat{z}, u_{O F C}\right) \\
& +B_{c} b(\hat{z}) T_{g o p t} \text { as } t \rightarrow \infty
\end{aligned}
$$

Finally, this will give the scientific interpretation for using semi-global output feedback control of nonlinear systems. Using inverse Park's transformation, gives $(\alpha, \beta)$ stationary reference frame $u_{O F C}$ of PMSG from (d-q) rotor frame as:

$u_{O F C}=\left[\begin{array}{c}\hat{u}_{g \alpha} \\ \hat{u}_{g \beta}\end{array}\right] \triangleq\left[\begin{array}{cc}\cos \hat{\theta}_{r e}-\sin \hat{\theta}_{r e} \\ \sin \hat{\theta}_{r e} & \cos \hat{\theta}_{r e}\end{array}\right]\left[\begin{array}{c}\hat{u}_{g d} \\ \hat{u}_{g q}\end{array}\right]$

It is more convenient, however, to express $u_{O F C}$ in the original coordinates before using change of variables, since the state feedback control is implemented in these coordinates.

Figure 3 clarifies the computer flowchart of the semiglobal output feedback MPPT control strategy for DDWTG system.

Lemma 1 [32] Let us consider the output of the system model and the continuous time output state predictor as specified in (78), (88), the output prediction error $e_{w}(t)$ can be re-written as:

$$
\begin{gathered}
\left\|e_{w}(t)\right\|^{2} \leq \theta(t)^{2 r}\left(\theta+\sqrt{q} \beta_{0}\right)^{2} T_{\max } \\
\int_{t-T_{\max }}^{t}\left\|\epsilon_{z}(s)\right\|^{2} d s-\left[C_{c} B_{c} T_{\text {gopt }}\right. \\
\left.\int_{t-T_{\max }}^{t}\|b(z(s))\| d s\right]^{2}
\end{gathered}
$$




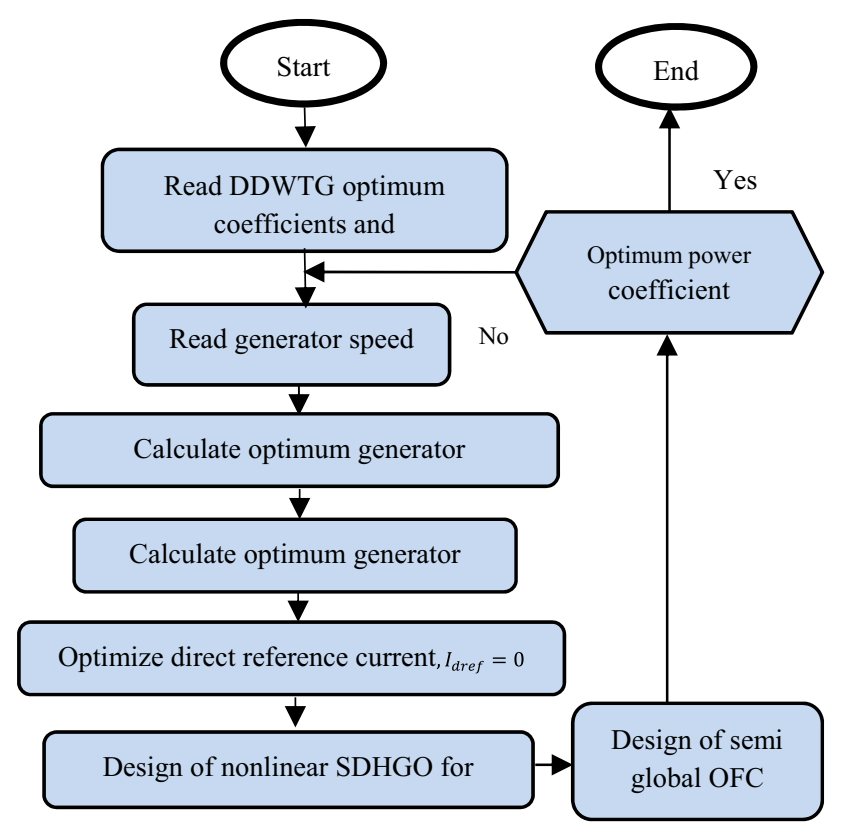

Fig. 3 Computer flowchart of the proposed approach

$\forall t \in\left[t_{k}, t_{k+1}\right), \mathrm{k} \in\{1, \ldots, s-1\}, q \in R, \beta_{0}>0$, Lipchitz constant. The maximum sampling time interval is denoted by $T_{\max }=\max _{k \in N}\left(t_{k+1}-t_{k}\right), \mathbb{N}$ is set of nonnegative integers.

Proof Notice that from (78) and (88), one gets the following:

$$
\begin{aligned}
\dot{e}_{w}= & \dot{w}(t)-\dot{y}(t)=C_{c}\left(A_{c} \hat{z}+\varphi\left(\operatorname{sat}(\hat{z}), u_{O F C}\right)\right) \\
& -C_{c}\left(A_{c} z+\varphi\left(\operatorname{sat}(z), u_{O F C}\right)+B_{c} b(z) T_{\text {gopt }}\right) \\
\dot{e}_{w}= & C_{c} A_{c} e_{z}+\varphi_{o 1}\left(\operatorname{sat}(\hat{z}), u_{O F C}\right) \\
& -\varphi_{1}\left(\operatorname{sat}(z), u_{O F C}\right)-C_{c} B_{c} b(z) T_{\text {gopt }} \\
& \forall \mathrm{t} \in\left[t_{k}, t_{k+1}\right), \mathrm{k} \in \mathbb{N}
\end{aligned}
$$

Thus, integrating the above equation for integration limits between $t_{k} \& t$, based on inter-sampled behavior, gives:

$$
\begin{aligned}
e_{w} & =\int_{t_{k}}^{t} C_{c} A_{c} e_{z} d s+\int_{t_{k}}^{t} \varphi_{1}\left(\operatorname{sat}(\hat{z}(s)), \text { uOFC }_{O}\right) \\
& -\varphi_{1}\left(\operatorname{satz}(s), \text { uOFC }_{O}\right) d s-\int_{t_{k}}^{t} C_{c} B_{c} b(z) T_{\text {gopt }} d s
\end{aligned}
$$

Then, using the principle change of coordinates, to simplify the mathematical calculations, as defined in (92) and using the identities: $\Delta A_{c} \Delta^{-1}=\theta A_{c}$ and $C_{c} \Delta=C_{c} \Delta^{-1}=C_{c}$, one gets:

$$
\begin{aligned}
\left\|e_{w}(t)\right\| \leq & \int_{t_{k}}^{t}\left\|C_{c} \Delta A_{c} \Delta^{-1} \theta^{r} \epsilon_{z}(s)\right\| d s \\
& +\int_{t_{k}}^{t} \| C_{c} \Delta \theta^{r}\left[\varphi_{o}\left(\operatorname{sat}(\hat{z}(s)), u_{O F C}\right)\right.
\end{aligned}
$$

$$
\begin{aligned}
& \left.-\varphi\left(\operatorname{sat}(z(s)), u_{O F C}\right)\right] \| d s \\
& -\int_{t_{k}}^{t}\left\|C_{c} B_{c} b(z) T_{\text {gopt }}\right\| d s
\end{aligned}
$$

Therefore, in view of hypothesis $\boldsymbol{H}_{\mathbf{1}}$, one can write:

$$
\begin{aligned}
\left\|e_{w}\right\| \leq & \int_{t_{k}}^{t}\left\|\theta^{r+1} C_{c} \epsilon_{z}(s)\right\| d s \\
& +\int_{t_{k}}^{t}\left\|\sqrt{q} \beta_{0} \theta^{r} \epsilon_{z}(s)\right\| d s \\
& -\int_{t_{k}}^{t}\left\|C_{c} B_{c} b(z) T_{\text {gopt }}\right\| d s \\
\left\|e_{w}\right\| \leq & \theta^{r}\left(\theta+\sqrt{q} \beta_{0}\right) \\
& \int_{t_{k}}^{t}\left\|\epsilon_{z}(s)\right\| d s-C_{c} B_{c} T_{\text {gopt }} \int_{t_{k}}^{t}\|b(z(s))\| d s
\end{aligned}
$$

Since, $T_{\max } \geq T_{s}, T_{s}=t_{k+1}-t_{k}, \forall k \in\{1, \ldots, s-1\}$

One confirms that:

$$
\begin{aligned}
\left\|e_{w}\right\| & \leq \theta^{r}\left(\theta+\sqrt{q} \beta_{0}\right) \int_{t-T_{\max }}^{t}\left\|\epsilon_{z}(s)\right\| d s \\
& -C_{c} B_{c} T_{\text {gopt }} \int_{t-T_{\max }}^{t}\|b(z(s))\| d s
\end{aligned}
$$

Pointing out Jensen`s inequality, this gives [36]:

$$
\left(\int_{t-T_{\max }}^{t}\left\|\epsilon_{z}(s)\right\|\right)^{2} d s \leq T_{\max } \int_{t-T_{\max }}^{t}\left\|\epsilon_{z}(s)\right\|^{2} d s
$$

So, one obtains the required mathematical formula of Lemma 1 after squaring both sides of (115). Let us consider the optimum generator torque function $T_{\text {gopt }}$ has:

$T_{\text {gopt }}$ is bounded and unknown periodic function, but there exist known function $\delta^{*}(t)>0$ such that $\left\|T_{\text {gopt }}\right\| \leq \delta^{*}(t)$ this implies, $\Delta B_{c}\left\|b(z) T_{\text {gopt }}\right\| \leq \frac{\beta_{1}}{\theta^{2}} \delta^{*}(t), \exists \beta_{1}>0$, is Lipchitz constant, yields

$$
\begin{aligned}
\left\|e_{w}\right\|^{2} & \leq \theta^{2 r}\left(\theta+\sqrt{q} \beta_{0}\right)^{2} T_{\max } \int_{t-T_{\max }}^{t}\left\|\epsilon_{z}(s)\right\|^{2} d s \\
& -C_{c}^{2}\left\|\frac{\beta_{1}}{\theta^{2}} \delta^{*}(t)\right\|^{2}
\end{aligned}
$$

Consequently, one introduced an explicit expression of continuous-time output prediction error between consecutive sampling instants depends on other sampled high-gain design parameters. 


\section{Stability analysis of the proposed sampled HGO design}

\section{Part I: Stability convergence analysis}

In order to prepare an upper bound solution of maximum admissible sampling period and to ensure the semi-global exponential convergence of the observation error towards zero, this study will re-formulate the theoretical results introduced by [32] in a more convenient and compatible for the case study in form of the Lyapunov-based analysis, which consists of direct Lyapunov function and Lyapunov Krasovskii functional LKF method for time dependent sampled-data systems:

$W_{0}\left(\epsilon_{z}\right) \triangleq \epsilon_{z}^{T} P \epsilon_{z}+\frac{1}{T_{\max }} \int_{t-T_{\max }}^{t} \int_{s}^{t}\left\|\epsilon_{z}\right\|^{2}(\xi) d \xi d s$

where $\mathrm{P}$ is a symmetric positive definite SPD matrix such that $P=P^{T} \geq 0, P \in R^{6 \times 6}$.

For some real constant $0<\sigma \leq 1, \sigma I_{n} \leq P \leq I_{n}$, therefore, $P$ satisfies the algebraic Lyapunov equation stated in (81). Let us split the proposed overall Lyapunov function into two functions named, $W_{1}$ and $W_{2}$, such that:

$$
\begin{aligned}
& W_{4}\left(\epsilon_{z}\right) \triangleq \epsilon_{z}^{T} P \epsilon_{z} \\
& W_{5}\left(\epsilon_{z}\right) \triangleq \frac{1}{T_{\max }} \int_{t-T_{\max }}^{t} \int_{s}^{t}\left\|\epsilon_{z}(\xi)\right\|^{2} d \xi d s
\end{aligned}
$$

The time derivative of the overall Lyapunov functions along the closed-loop trajectory of (98) in (118) and (119) is given by:

$\dot{W}_{0}\left(\epsilon_{z}\right)=\dot{W}_{4}\left(\epsilon_{z}\right)+\dot{W}_{5}\left(\epsilon_{z}\right)$

One still use, $\dot{W}_{4}\left(\epsilon_{z}\right)=\epsilon_{z}^{T} P \dot{\epsilon}_{z}+\dot{\epsilon}_{z}^{T} P \epsilon_{z}$

Let us use the variable, $t_{0} \in R$, within the integration limits of (119), one can re-write (119) in new form as:

$$
\begin{aligned}
& W_{5}\left(\epsilon_{z}\right) \triangleq \int_{t_{0}}^{t}\left\|\epsilon_{z}(\xi)\right\|^{2} d \xi \\
& -\frac{1}{T_{\max }} \int_{t-T_{\max }}^{t} \int_{t_{0}}^{s}\left\|\epsilon_{z}(\xi)\right\|^{2} d \xi d s
\end{aligned}
$$

So, the time derivative of the fifth Lyapunov functions along the closed-loop trajectory of (99) is:

$\dot{W}_{5}\left(\epsilon_{z}\right)=\left\|\epsilon_{z}\right\|^{2}-\frac{1}{T_{\max }} \int_{t-T_{\max }}^{t}\left\|\epsilon_{z}(s)\right\|^{2} d s$
In view of (98), one can calculate the time derivative of the fourth Lyapunov function along the closed loop trajectory as:

$$
\begin{aligned}
\dot{W}_{4}= & \theta \epsilon_{z}^{T}\left[\left(A_{c}-K C_{c}\right)^{T} P\right] \epsilon_{z} \\
& +\frac{2}{\theta^{r}} \epsilon_{z}^{T} P \Delta\left\{\varphi_{o}\left(\operatorname{sat}(\hat{z}), u_{O F C}\right)-\varphi\left(\operatorname{sat}(z), u_{O F C}\right)\right\} \\
& +\frac{2}{\theta^{r-1}} \epsilon_{z}^{T} P K e_{w}-\frac{2}{\theta^{r}} P \epsilon_{z}^{T} B_{c} b(z) T_{g o p t} \\
& -2 \frac{\dot{\theta}}{\theta} \epsilon_{z}^{T} P D \epsilon_{z}
\end{aligned}
$$

In view of algebraic Lyapunov function stated in (81) to choose $P$ and the definition of diagonal matrix stated in (79), the time derivative of fourth Lyapunov function in (122):

$$
\begin{aligned}
& \dot{W}_{4}\left(\epsilon_{z}\right) \leq-\left(\theta \mu+2 r \frac{\dot{\theta}}{\theta}\right) I_{6} W_{1}\left(\epsilon_{z}\right) \\
& +\frac{2}{\theta^{r}} \epsilon_{z}^{T} P \Delta\left\{\varphi_{o}\left(\operatorname{sat}(\hat{z}), u_{O F C}\right)-\varphi\left(\operatorname{sat}(z), u_{O F C}\right)\right\} \\
& +\frac{2}{\theta^{r-1}} \epsilon_{z}^{T} P K e_{w}-\frac{2}{\theta^{r}} P \epsilon_{z}^{T} B_{c} b(z) T_{g o p t}-\frac{2 \theta}{\theta} \epsilon_{z}^{T} P E \epsilon_{z}
\end{aligned}
$$

As well as the strictly positive real number $r>1$, is chosen such that it satisfies the following linear matrix inequality [32]:

$-r P \leq P E+E P \leq r P$

Thus, according to (124), the last term of (123) becomes:

$$
\begin{aligned}
& -2 \frac{\dot{\theta}}{\theta} \epsilon_{z}^{T} P E \epsilon_{z} \leq r \frac{|\dot{\theta}|}{\theta} \epsilon^{T} P \epsilon_{z} \\
& \dot{W}_{4}\left(\epsilon_{z}\right) \leq-\left(\theta \mu+2 r \frac{\dot{\theta}}{\theta}-r \frac{|\dot{\theta}|}{\theta}\right) W_{1}\left(\epsilon_{z}\right) \\
& +\frac{2}{\theta^{r}} \epsilon_{z}^{T} P \Delta\left\{\varphi_{o}\left(\operatorname{sat}(\hat{z}), u_{O F C}\right)-\varphi\left(\operatorname{sat}(z), u_{O F C}\right)\right\} \\
& +\frac{2}{\theta^{r-1}} \epsilon_{z}^{T} P K e_{w}-\frac{2}{\theta^{r}} P \epsilon_{z}^{T} B_{c} b(z) T_{\text {gopt }}
\end{aligned}
$$

In view of first inductive hypothesis $\boldsymbol{H}_{\mathbf{1}}$ for locally Lipschitz function, one deduces that:

$$
\begin{aligned}
& \dot{W}_{4}\left(\epsilon_{z}\right) \leq-\left[\theta \mu+2 r \frac{\dot{\theta}}{\theta}-r \frac{|\dot{\theta}|}{\theta}-2 \sqrt{q} \beta_{0} \frac{\lambda \max (P)}{\lambda \min (P)}\right] \\
& \times W_{1}\left(\epsilon_{z}\right) \\
& +\frac{2}{\theta^{r-1}} \epsilon_{z}^{T} P K e_{w}-\frac{2}{\theta^{r}} \epsilon_{z}^{T} P B_{c} b(z) T_{g o p t}
\end{aligned}
$$

Using the well-known inequality called Young's inequality, let us choose $\mathrm{X}=\mathrm{Y}=2 \rightarrow \frac{1}{X}+\frac{1}{Y}=1$, that is $\frac{a^{2}}{\vartheta}+\vartheta b^{2} \geq$ $a b, \forall \vartheta>0$, one shall choose, $\vartheta=\frac{\mu \theta \lambda \min (P)}{2}$, this implies: 


$$
\begin{aligned}
& \dot{W}_{4}\left(\epsilon_{z}\right) \leq-\left[\frac{\theta}{2} \mu+2 r \frac{\dot{\theta}}{\theta}-r \frac{|\dot{\theta}|}{\theta}-2 \sqrt{q} \beta_{0} \frac{\lambda \max (P)}{\lambda \min (P)}\right] \\
& W_{1}\left(\epsilon_{z}\right)+\frac{2 \lambda \max ^{2}(P)}{\theta^{2 r-2} \mu \lambda \min (P)} \\
& \|K\|^{2}\left\|e_{w}\right\|^{2}-\frac{2}{\theta^{r}} \epsilon_{z}^{T} P \Delta B_{c} b(z) T_{\text {gopt }}
\end{aligned}
$$

According to Lemma 1, it leads us to get the following expression for Lyapunov equation $\forall t \in\left[t_{k}, t_{k+1}\right)$ :

$$
\begin{gathered}
\dot{W}_{4}\left(\epsilon_{z}\right) \leq-\left[\frac{\theta}{2} \mu+2 r \frac{\dot{\theta}}{\theta}-r \frac{|\dot{\theta}|}{\theta}-2 \sqrt{q} \beta_{0} \frac{\lambda \max (P)}{\lambda \min (P)}\right] \\
W_{1}\left(\epsilon_{z}\right)+T_{\max } \theta^{2 r}\left[\theta+\sqrt{q \beta_{0}}\right]^{2} \\
\left(\frac{2 \lambda \max ^{2}(P)}{\theta^{2 r-1} \mu \lambda \min (P)}\|K\|^{2} \int_{t-T_{\max }}^{t}\left\|\epsilon_{z}(s)\right\|^{2} d s\right. \\
-\frac{2}{\theta^{r}} \epsilon_{z}^{T} P \Delta B b(z) T_{\text {gopt }}
\end{gathered}
$$

Adding the time derivative of Lyapunov functions stated in (122) and (128), respectively, which will give the overall time derivative of Lyapunov function is: $\dot{W}_{0}\left(\epsilon_{z}\right)=\dot{W}_{4}\left(\epsilon_{z}\right)+$ $\dot{W}_{5}\left(\epsilon_{z}\right)$

$$
\begin{aligned}
& \dot{W}_{0}\left(\epsilon_{z}\right)=-\left[\frac{\theta}{2} \mu+2 r \frac{\dot{\theta}}{\theta}-r \frac{|\dot{\theta}|}{\theta}-2 \sqrt{6} \beta_{0} \frac{\lambda \max (P)}{\lambda \min (P)}\right] W_{1}\left(\epsilon_{z}\right) \\
& \left.+T_{\max }\left[\theta+\sqrt{6} \beta_{0}\right]\right)^{2} \frac{2 \theta \lambda \max ^{2}(P)}{\mu \lambda \min (P)}\|K\|^{2} \\
& \int_{t-T_{\max }}^{t}\left\|\epsilon_{z}(s)\right\|^{2} d s-+\frac{2}{\theta^{r}} \epsilon_{z}^{T} P \Delta B_{c} b(z) T_{g o p t} \\
& -C_{c}^{2}\left\|\frac{\beta_{1}}{\theta^{2}} \bar{\delta}\right\|^{2}+\left[\left\|\epsilon_{z}\right\|^{2}-+\frac{1}{T_{\max }} \int_{t-T_{\max }}^{t}\right. \\
& \left.\int_{s}^{t}\left\|\epsilon_{z}(s)\right\|^{2} d s\right]
\end{aligned}
$$

\section{Part II: Derivative of maximum allowable sampling} period

Let us bound the parameter $q=6$, for the sample study of sixth order state-space representation.

$$
\begin{aligned}
& \dot{W}_{0}\left(\epsilon_{z}\right) \leq-\left[\frac{\theta}{2} \mu+2 r \frac{\dot{\theta}}{\theta t}-r \frac{|\dot{\theta}|}{\theta t}-2 \sqrt{6} \beta_{0} \frac{\lambda \max (P)}{\lambda \min (P)}\right] \\
& W_{1}\left(\epsilon_{z}\right)-\left[\frac{1}{T_{\max }}-T_{\max } \frac{2 \theta \lambda \max ^{2}(P)}{\mu \lambda \min (P)}\|K\|^{2}\left(\theta+\sqrt{6} \beta_{0}\right)\right]^{2} \\
& \int_{t-T_{\max }}^{t}\left\|\epsilon_{z}(s)\right\|^{2} d s-\frac{2}{\theta^{r}} \epsilon_{z}^{T} P \Delta B_{c} b(z) T_{\text {gopt }} \\
& -C_{c}^{2}\left\|\frac{\beta_{1}}{\theta^{2}} \bar{\delta}\right\|^{2}
\end{aligned}
$$

To create semi-global exponential convergence of the observation error under output feedback nonlinear control and to ensure that the time derivative of the overall Lyapunov function given in (130) is negative, this study will introduce an explicit expression for upper bound of sampling interval depends on other design parameters as follows:

$\left[\frac{1}{T_{\max }}-T_{\max } \frac{2 \theta \lambda \max ^{2}(P)}{\mu \lambda \min (P)}\|K\|^{2}\left(\theta+\sqrt{6} \beta_{0}\right)^{2}\right] \geq 0$

Finally, taking root mean square for both sides, $\mathrm{q}=6$, and $\theta \rightarrow \theta_{\max }$, thus, $\forall \theta_{\max } \geq \theta\left(t_{0}\right)>1$, and $\forall T_{s} \in\left(0, T_{\max }\right]$ is piecewise function, yields:

$T_{\max } \leq \frac{\sqrt{\mu \lambda \min (P)}}{\sqrt{2 \theta_{\max }} \lambda \max (P)\|K\|\left(\theta_{\max }+\sqrt{6} \beta_{0}\right.}$

where, $\theta_{\max }$ is the maximum amplitude of the high gain design parameter, $\forall t \in R^{+}, \beta_{0}>0$ and $\lambda_{\min }(P), \lambda_{\max }(P)$ are respectively the largest and smallest eigenvalues of the positive definite matrix P. Using the following property:

$\lambda \min (P)\left\|\epsilon_{z}(s)\right\|^{2} \leq W_{1}\left(\epsilon_{z}\right) \leq \lambda \max (P)\left\|\epsilon_{z}\right\|^{2}$, and one shall get after substituting (131) in (130),

$$
\begin{gathered}
\dot{W}_{0}\left(\epsilon_{z}\right) \leq-\left[\frac{\theta}{2} \mu+2 r \frac{\dot{\theta}}{\theta}-r \frac{|\dot{\theta}|}{\theta}-2 \sqrt{6} \beta_{0} \frac{\lambda_{\max }(P)+1}{\lambda_{\min }(P)}\right] \\
W_{1}\left(\epsilon_{z}\right)-+\frac{2}{\theta^{r}} \epsilon_{z}^{T} P \Delta B_{c} b(z) T_{\text {gopt }}-C_{c}^{2}\left\|\frac{\beta_{1}}{\theta^{2}} \delta^{*}(t)\right\|^{2}
\end{gathered}
$$

As the dynamic HGO design parameter is increasing in the discrete time interval $\left[t_{k}, t_{k+1}\right)$, let us select the dynamic high-gain observer design parameter $\dot{\theta}$ or $g(\theta), g: R \rightarrow R$ function describing the observer design parameter with the adaptation process is driven by assessment of the system model nonlinearities that will guarantee to enlarge the sampling period with fast convergence:

$\dot{\theta} \triangleq-\frac{\theta}{r}\left[\frac{\mu}{2} \theta+2 r \frac{\dot{\theta}+|\dot{\theta}| \beta_{0} \lambda \max (P)}{\theta \lambda \min (P)}\right]$

The adaptation is driven by assessment of the system model nonlinearities.

To summarize the proposed main theorem, using Lyapunov stability approach of nonlinear systems, one concludes that, with the choice for the gain matrix corresponding to measurements error $K$ stated in (80) and it submitted to algebraic Lyapunov equation in (81), the DHGO design parameter $\dot{\theta}=g(\theta)$ proposed in (133) and explicitly introduce an upper bound of positive sampling period $T_{\max }$ stated in (131) supported by semi-global sampled HGO observer defined in (84), $\forall t \in\left[t_{k}, t_{k+1}\right), \mathrm{k} \in \mathbb{N}$, as well as introduce an explicit expression for output prediction error for time dependent of sampled systems in terms of other design parameters for whatever initial state variables stated in (116) and confirming 


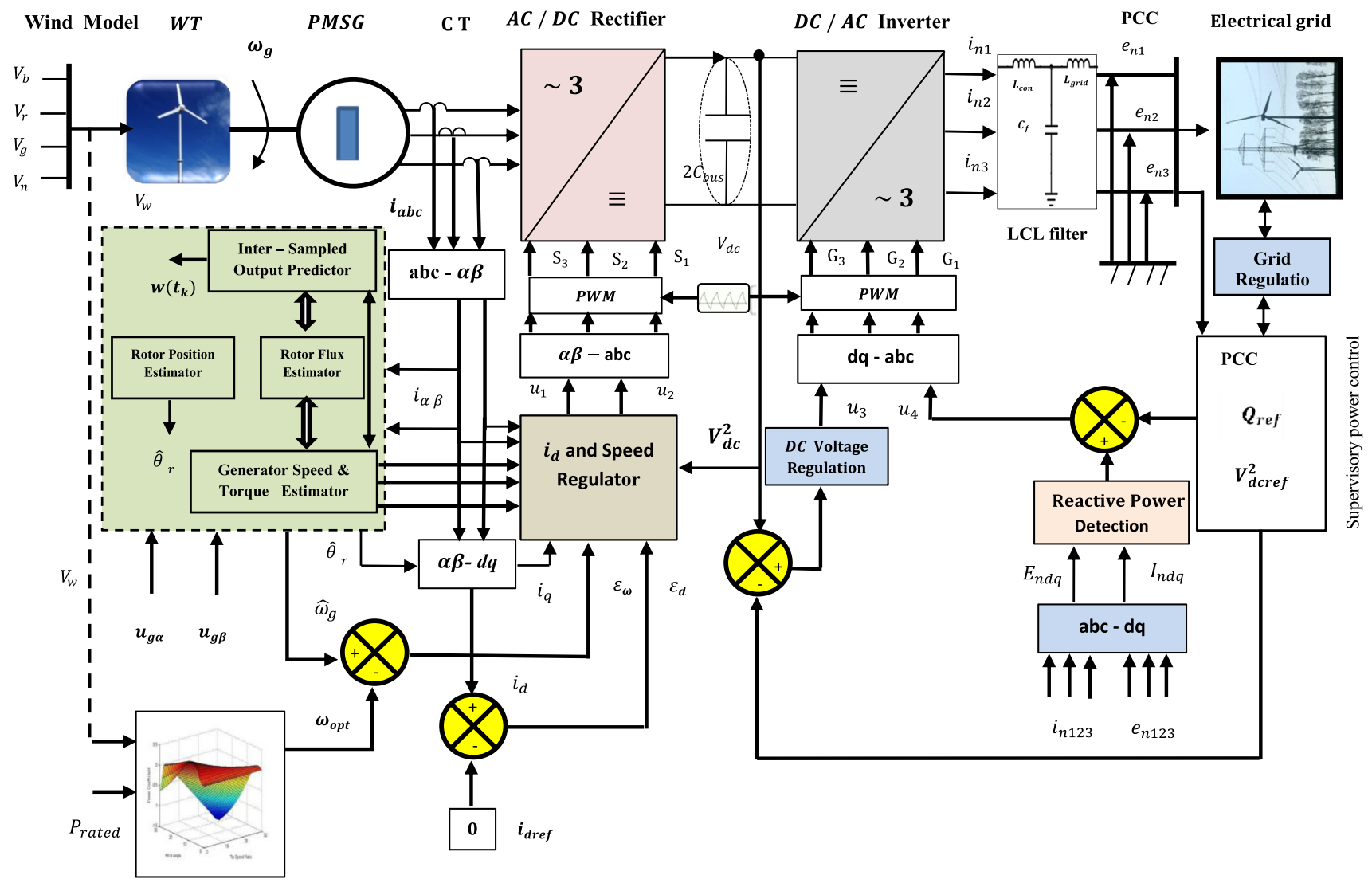

Fig. 4 Output feedback control diagram of WTG system coupled with nonlinear sampled-HGO design

for HGO that $\theta\left(t_{0}\right)>1$. This theorem guarantees globally exponential convergence of observer (84) towards its real system of (74).

Remark 3 From all shown above and according to all time derivative candidate Lyapunov functions stated in (19), (29), (121) and (123) one ensure the semi-global exponential convergence SGES of the proposed observer and the exponential vanish of the observation error under semi-global output feedback control of closed-loop dynamic system defined by (100), (101), (102) and (103) using Lyapunov stability approach. This completes proof of theorem 1

\section{Numerical simulation and verifications}

\subsection{Implementation of semi-global sampled HGO design}

In this subsection, the dynamic performances of the proposed semi-global sampled high-gain observer having dynamic design parameter for online estimation of wind turbine generator system state variables, which are generator rotor position, rotor speed and unknown bounded input generator torque. DDWTG system has been implemented using MATLAB/Simulink environment (V.R2015a). The tool selected for solving the dynamic equations is the MATLAB function called ODE45 with relative tolerance of $2 * 10^{-5}$.

The whole control block diagram for back to back PWM converter association connected together with DC power transfer link equipped with DDWTG system is demonstrated in Fig. 4.

Rotor speed tracking error, $\varepsilon_{\omega}$ and tracking error, $\varepsilon_{d}$ related with $d$-axis current regulator accomplished by backstepping controller are used to derive first and second practical control law used for the three-phase PWM control of generator-side converter. Similarly for grid-side converter, dynamic tracking performance of DC link squared voltage error $\varepsilon_{V d c}$ accomplished by backstepping controller and reactive power tracking error $e_{n q}$ are used to derive third and fourth practical control law used for the three-phase PWM control of grid-side converter. The system dynamics of WTG have been described with sixth order nonlinear state-space model. Sampled HGO of system (84) is proposed to obtain online estimates of all mechanical state variables.

The sampled HGO design parameters are $\theta, T_{s}$ and $K$. Off course, the dynamic performance depends on the numerical values given to the observer parameters, $\theta, T_{s}$ and $K$. It is very necessary to emphasize that this study has been used the estimated value of electrical rotor position $\hat{\theta}_{e}$ for estimation other unmeasured mechanical state variables instead of using 
Table 2 Nominal specifications of the simulated DDWTG system

\begin{tabular}{ll}
\hline Electrical networks & $\begin{array}{l}\text { Grid voltage: } 400-230 \mathrm{~V} \\
\text { Grid frequency: } 50 \mathrm{~Hz},\end{array}$ \\
\hline AC/DC/AC PWM & $\begin{array}{l}L_{\text {grid }}=5.7 \mathrm{mH} ; L_{\text {conv }}=17.7 \mathrm{mH}, \\
\text { Converters }\end{array}$ \\
$\begin{array}{l}C_{\text {bus }}=25 \mathrm{mF}, C_{f}=3.45 \mu \mathrm{F} ; f_{\text {cut }- \text { off }}=3.9 \mathrm{kHz}, \text { converter switching frequency }=15 \mathrm{kHz}, \text { converter } \\
\text { efficiency }=85 \%\end{array}$ \\
Number of aerodynamic blades is 3, Radius of the rotor is $3 \mathrm{~m}$ \\
Wind turbine generator & Wind speed range $=(4-12) \mathrm{m} / \mathrm{s}, \mathrm{c}_{1}=0.5, \mathrm{c}_{2}=116, \mathrm{c}_{3}=0.2, \mathrm{c}_{4}=5, \mathrm{c}_{5}=21, \mathrm{c}_{6}=0.0068$, \\
& Wind density $=1.25 \mathrm{~kg} / \mathrm{m}^{3}$
\end{tabular}

Table 3 Feedback controller and observer design parameters

\begin{tabular}{lllr}
\hline Index & Value & Index & Value \\
\hline$k_{11}$ & 6 & $k_{12}$ & 6 \\
$k_{21}$ & 14 & $k_{22}$ & 1 \\
$k_{31}$ & 16 & $k_{32}$ & 3 \\
$\theta$ & Unknown & $h_{1}$ & 220 \\
$h_{2}$ & 50 & $h_{3}$ & 40 \\
$h_{4}$ & 50 & $h_{5}$ & 1000 \\
$h_{6}$ & 500 & & \\
\hline
\end{tabular}

Table 4 Numerical values of the maximum allowable sampling

\begin{tabular}{|c|c|}
\hline $\begin{array}{l}\text { Maximum allowable sampling } \\
\text { period and frequency }\end{array}$ & $\begin{array}{l}\text { Dynamic gain } \\
\dot{\theta}=g(\theta)\end{array}$ \\
\hline$T_{\max }$ & $T_{s 1}=4 \mathrm{~ms}$ \\
\hline$f_{s}$ & $250 \mathrm{~Hz}$ \\
\hline
\end{tabular}

rotor position encoder. Actual nominal numerical values of the DDWTG system are listed in Table 2.

The observer gain matrix corresponding to measurement error and other output feedback controller design parameters are summarized in Table 3, which proved to be suitable and sufficient for the sample study. Table 4. Numerical values of the maximum allowable sampling period and sampling frequency for fixed and dynamic high gain design parameters of the proposed sampled high gain observer. The initial pitch angle $\theta_{\text {pitch }}$ retains in the value of zero degree; the tip speed ratio maintains the best value of (8.2) as well as best power coefficient of (0.48). The variation of wind velocity

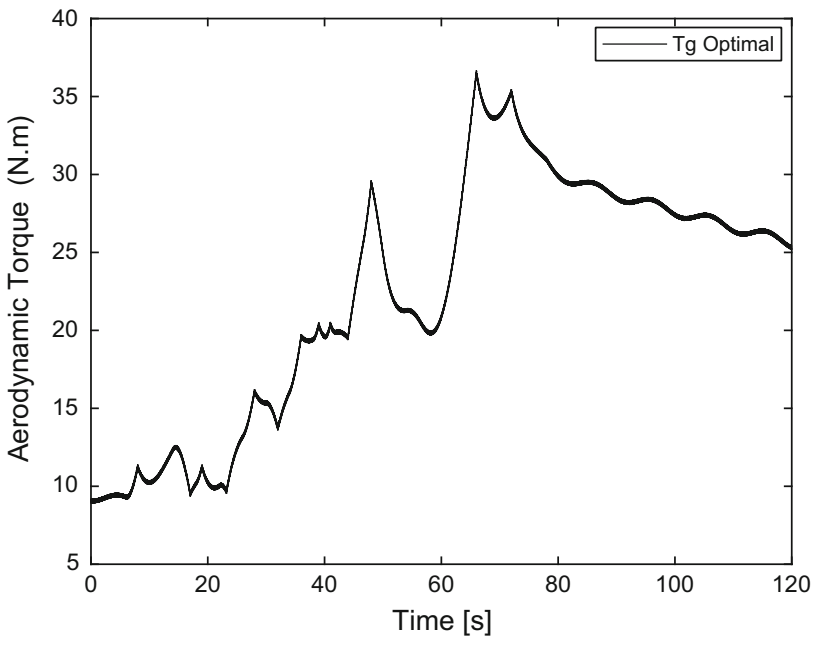

Fig. 5 Optimal generator torque $(\mathrm{Nm})$

and its optimized amplitude is clarified in Fig. 5 and the input mechanical torque profile is shown in Fig. 6.

Therefore, various simulations tests were conducted and their control objectives are the output feedback control of variable speed DDWTG system. Figures 7 and 8 are representing the best generator rotor speed (rad/s) and mechanical generated power in $(\mathrm{kW})$ taking into consideration influence of wind acceleration and turbulence on dynamic performance of WTG. Figure 10 illustrates dynamic high-gain design parameter of the proposed observer. The dynamical continuous-time design parameter is increasing in the time interval $\left[t_{k}, t_{k+1}\right), \mathbb{N}$ is set of nonnegative integers (Fig. 9).

In fact, the wind velocity is variable with time and fluctuated with influence of wind acceleration and turbulence, a variety of control mechanisms are employed to manage the 


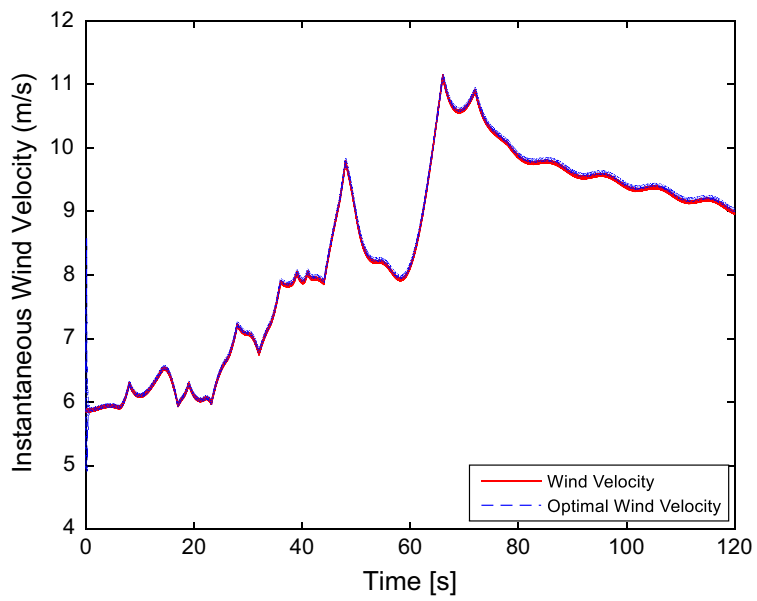

Fig. 6 Wind velocity and its optimal $(\mathrm{m} / \mathrm{s})$

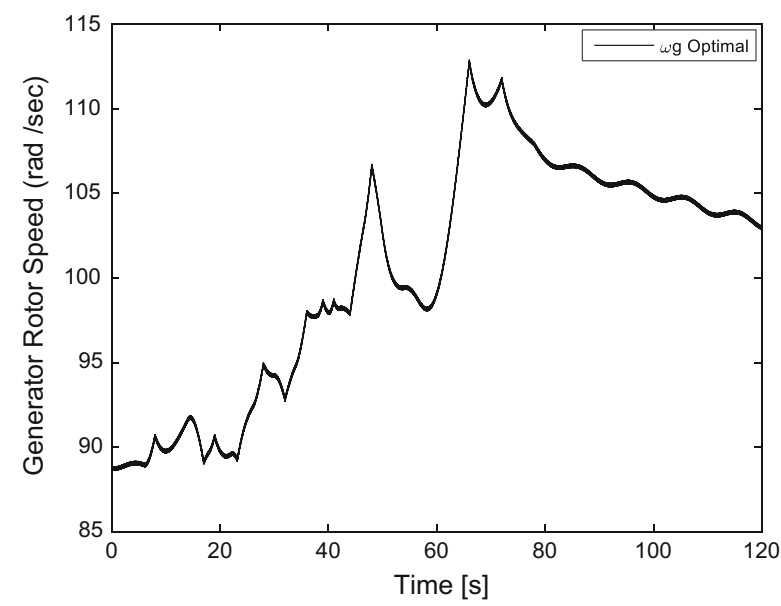

Fig. 7 Optimal generator rotor speed $(\mathrm{rad} / \mathrm{s})$

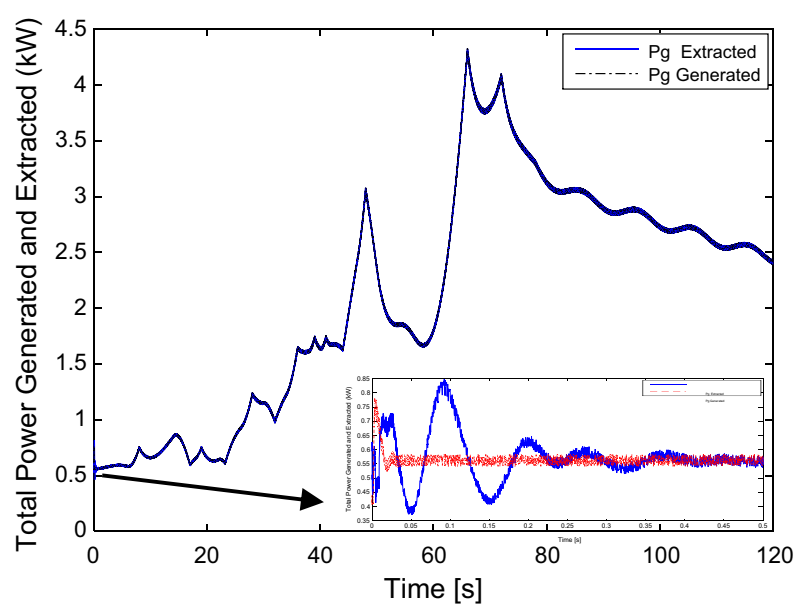

Fig. 8 Mechanical generated power $(\mathrm{kW})$

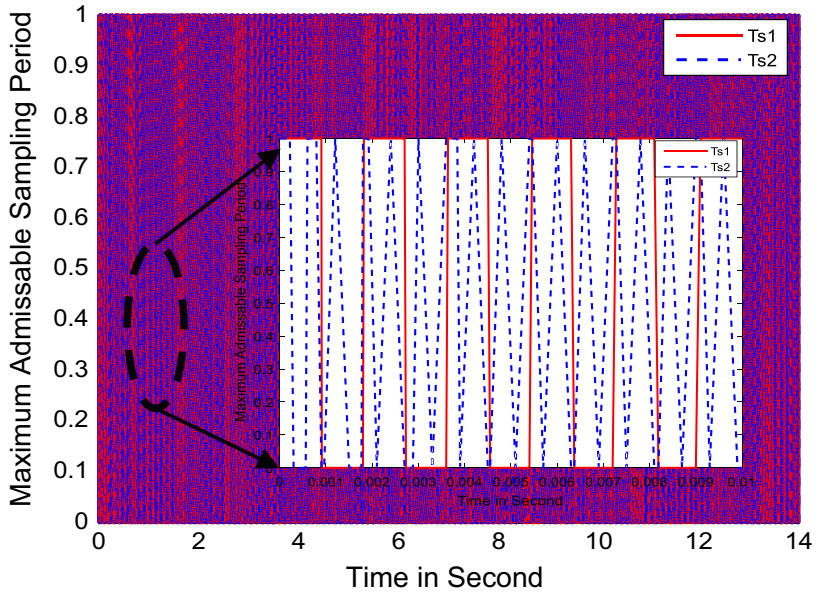

Fig. 9 Waveform of $T_{M}=t_{k+1}-t_{k}, \mathrm{k} \in \mathbb{N}$, for dynamic and fixed HGO design parameter

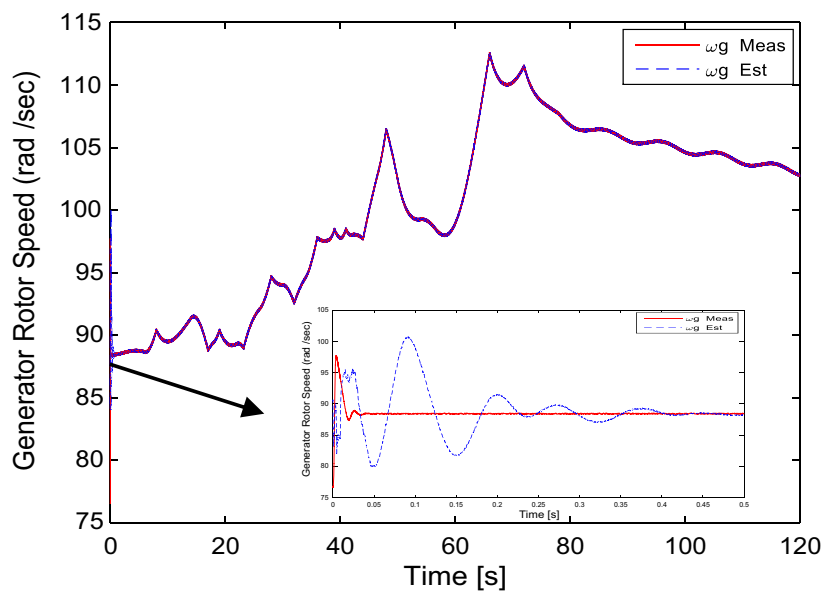

Fig. 10 Tracking of actual speed and its estimate $\hat{\omega}_{g}$

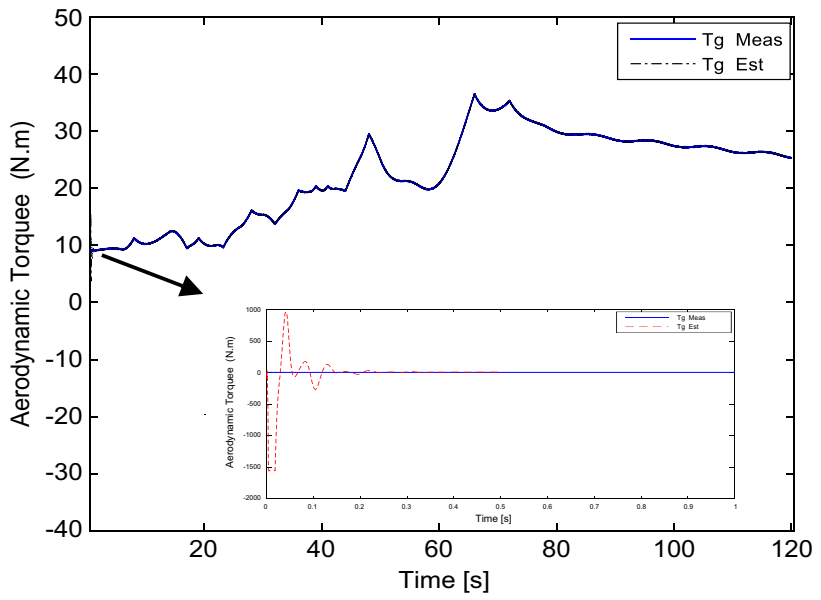

Fig. 11 Tracking performance of real torque $T_{g}$, observed torque $\hat{T}_{g}(\mathrm{Nm})$ 


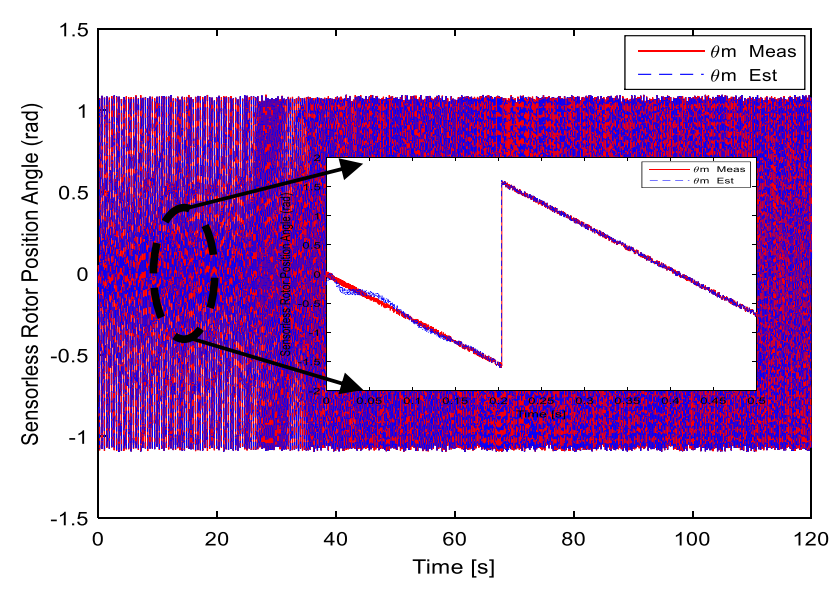

Fig. 12 Tracking performance of measured rotor angle $\theta_{r}$, observed rotor angle $\hat{\theta}_{r}(\mathrm{rad})$

conversion process, detect and protect the mechanical and electrical equipments from conditions that would result in failure or destruction.

The observer dynamic performances for complete cycle of (120 s) are shown in Figs. 10, 11, 12, 13 and 14 to clarify the dynamic tracking performance of the proposed sampled HGO is quite satisfactory for all DDWTG full order estimated variables at $T_{\max }$ of (6) ms. One can notice from Figs. 6 and 10 , that the observed generator rotor speed inter the second stable region of operation for DDWTG system after $0.5 \mathrm{~s}$. For this moment, it is observed that the closed-loop trajectories of proposed observer tend to their real system trajectories faster than the static gain observer trajectories, because the block diagonal matrix denoted by, $\Delta$, will be updated, simultaneously with dynamical high-gain design parameter, $\theta$ this means that the innovation correction term in proposed observer structure will be modified faster than the same term in comparative with static HGO design parameter, as the dynamical $\mathrm{HGO}$ design parameter is increasing in the time interval $\left[t_{k}, t_{k+1}\right)$. The adaptation is driven by assessment of the system model nonlinearities and HGO design parameters.

It is clearly noticeable from Figs. 13 and 14, the output generator currents in $(\alpha-\beta)$ reference frame and their output predictor are decreased and increased simultaneously with variation of best wind velocity and consequently with input mechanical generator profile as demonstrated in Figs. 5 and 6 , respectively. It is obvious from simulation results of case study that the startup wind velocity; $5.8 \mathrm{~m} / \mathrm{s}$, must be greater than cut-in wind velocity; $(4 \mathrm{~m} / \mathrm{s})$ to overcome the large starting aerodynamic torque and viscus friction. This problem is specially found in small-scale DDWTG with the initial pitch angle keeps the value of zero degree.

For reason that the given DDWTG system model does not have the Lipschitz property given in $\boldsymbol{H}_{\mathbf{1}}$, some of the saturation actuator functions given in (87) are introduced for

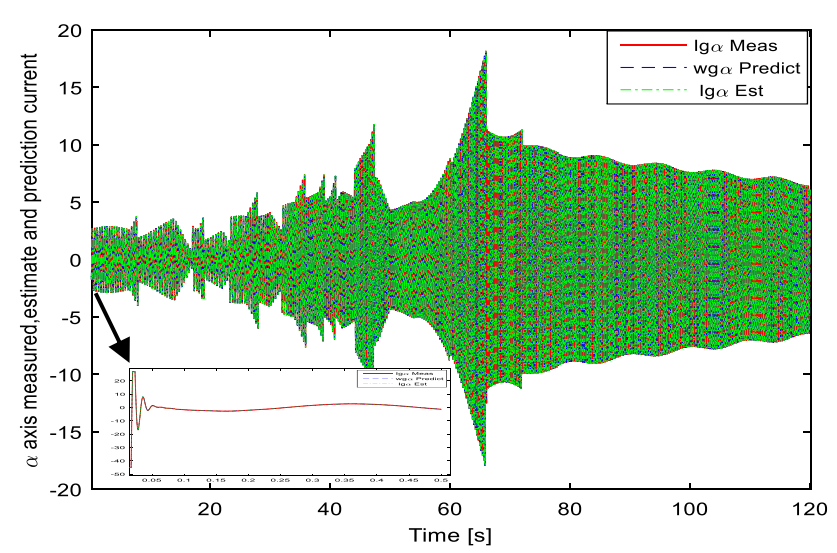

Fig. 13 Measured current $i_{g \alpha}$, predicted current $w_{g \alpha}$ and estimated $\hat{\iota}_{g \alpha}$ (A)

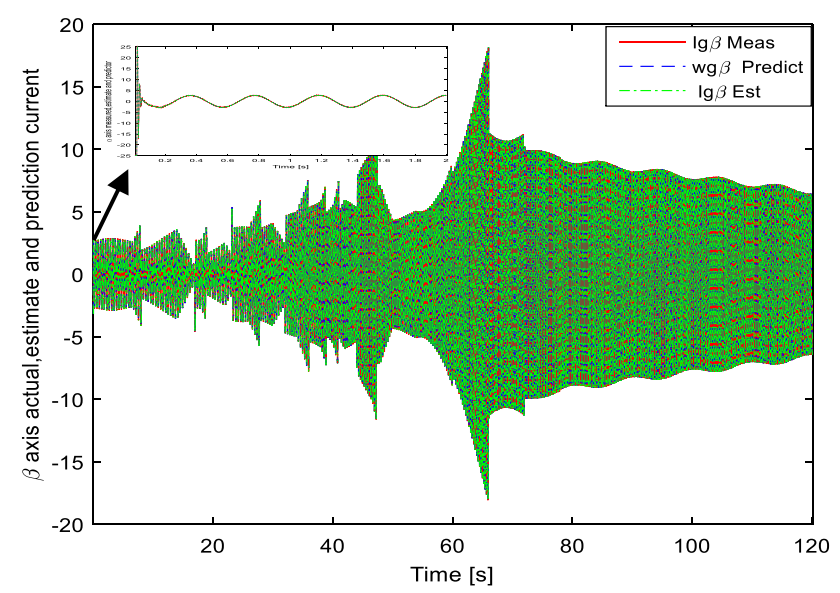

Fig. 14 Measured current $i_{g \beta}$, predicted current $w_{g \beta}$ and estimated $\hat{\iota}_{g \beta}$ (A)

the Lipschitz extension property in multi-input multi output MIMO system. As clarified in Figs. 13 and 14, the generator output current is bounded between $\{-20,20\} \mathrm{A}$.

\subsection{Implementation of semi-global output feedback controller}

The semi-global output feedback control strategy for this application is an approach based on nonlinear backstepping design technique accompanied by benefits of sensorless measurements. The semi-global output feedback control performances illustrated by the curves of Figs. 15, 16, 17, show that the best reference, measured and estimated rotor speed, $\omega_{g}$ and the direct axis component of the stator current, $i_{d}$ sufficiently converge to their respective references after small transient duration time in less than (0.5) s. Off course, generator rotor speed tracking and direct-axis stator current accomplished by backstepping design technique is necessary as stated and explained in section three for regulating generator rotor speed, to ensure that the reluctance torque of 


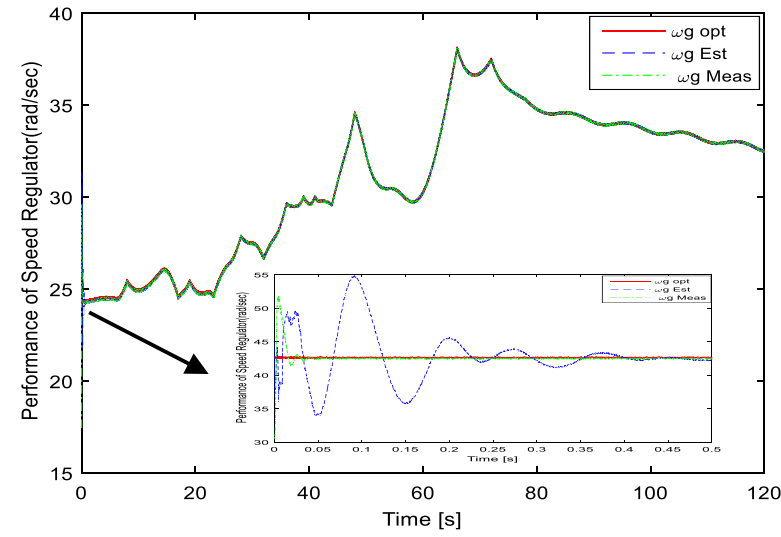

Fig. 15 Tracking performance of measured speed $\omega_{g}$ and observed speed $\hat{\omega}_{g}$ and its reference

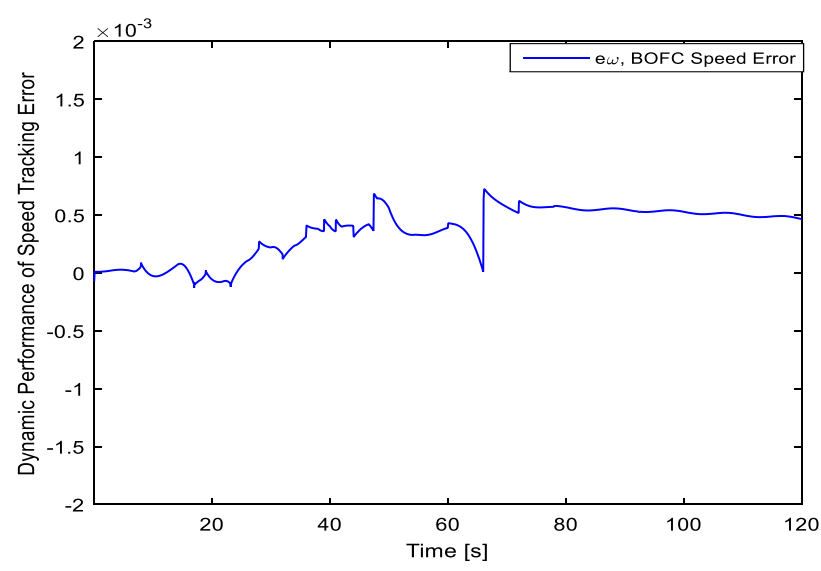

Fig. 16 Tracking of rotor speed error $\varepsilon_{\omega}$ under BOFC accomplished by Backstepping technique

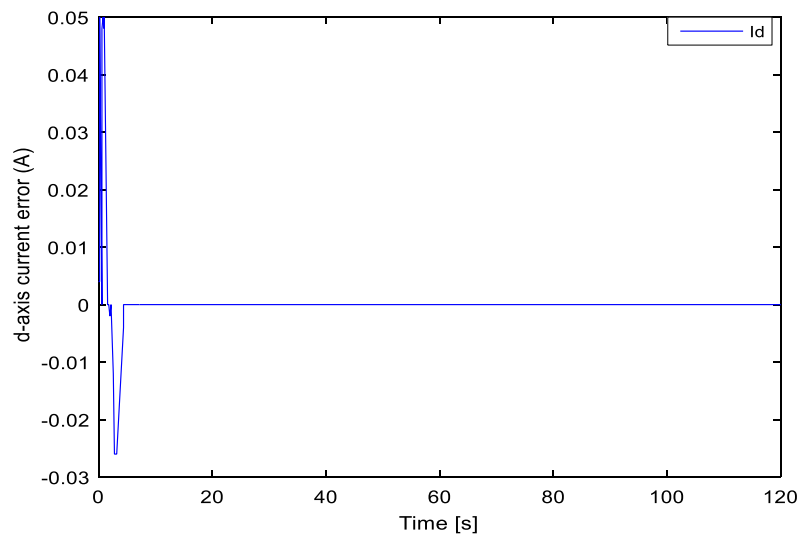

Fig. 17 Tracking error $\varepsilon_{d}$ for d-axis current under BOFC accomplished by Backstepping technique

PMSG is null and to optimize the generator stator current as small as possible based on the strategy of vector control (Fig. 18).

The semi-global output-feedback controller defined by (100), (101), (102), and (103) are simulated as shown in

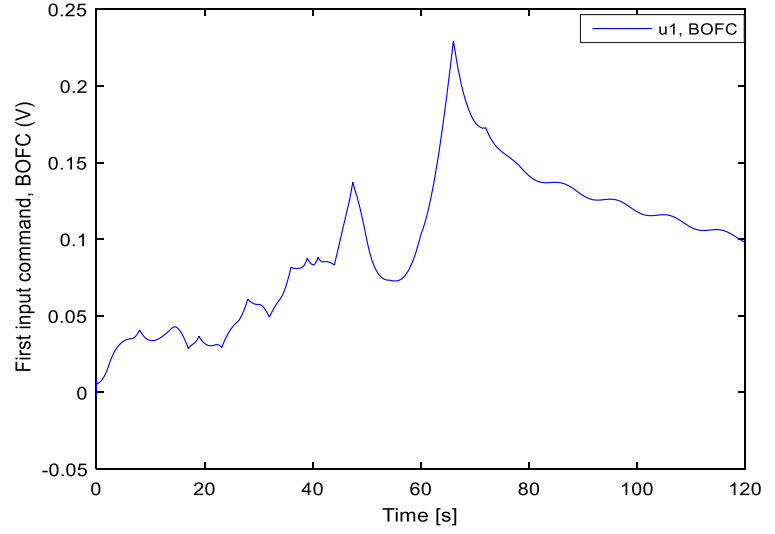

Fig. 18 First input command $u_{1}$ under BOFC accomplished by Backstepping technique

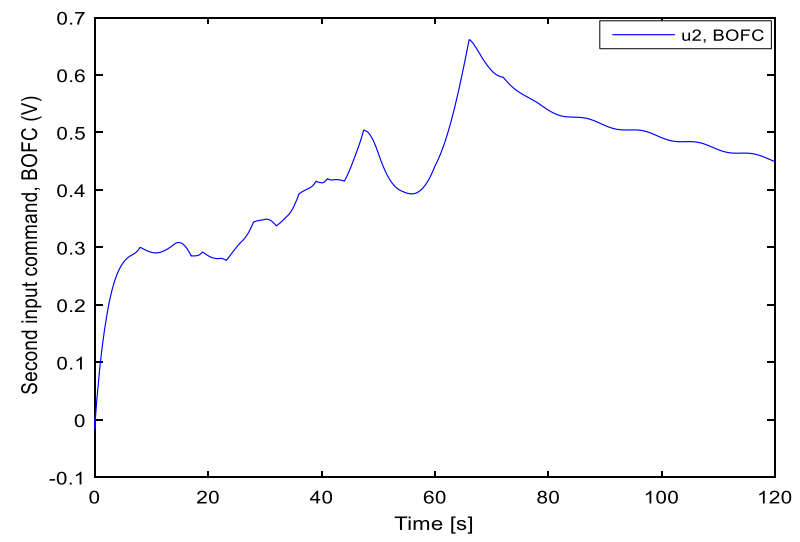

Fig. 19 Second input command $u_{2}$ under BOFC accomplished by Backstepping technique

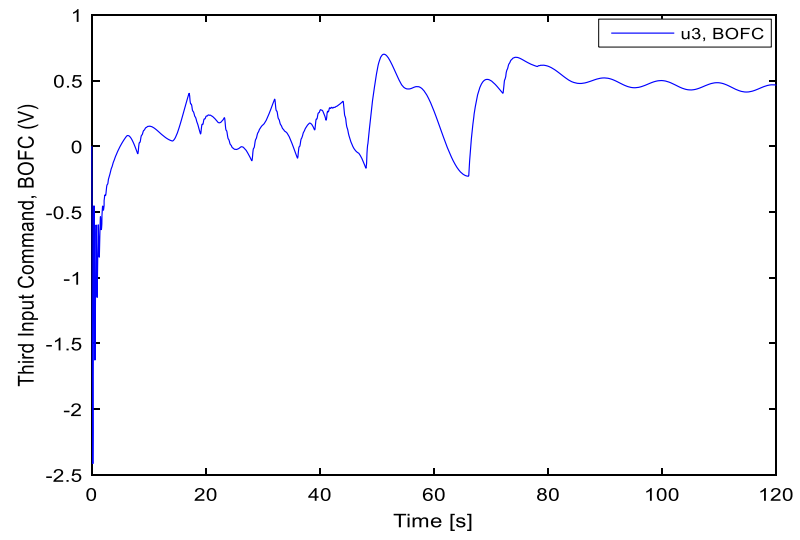

Fig. 20 Third input command $u_{3}$ under BOFC accomplished by Backstepping technique

Figs. 19, 20, 21, and 22, which are bounded between $\{-1,1\}$. The tracking of DC link voltage that considered $500 \mathrm{~V}$ across the shunt capacitor between generator side converter and grid-side converter is shown in Fig. 23, which satisfies the permissible voltage regulation range of $-2 \%$. 


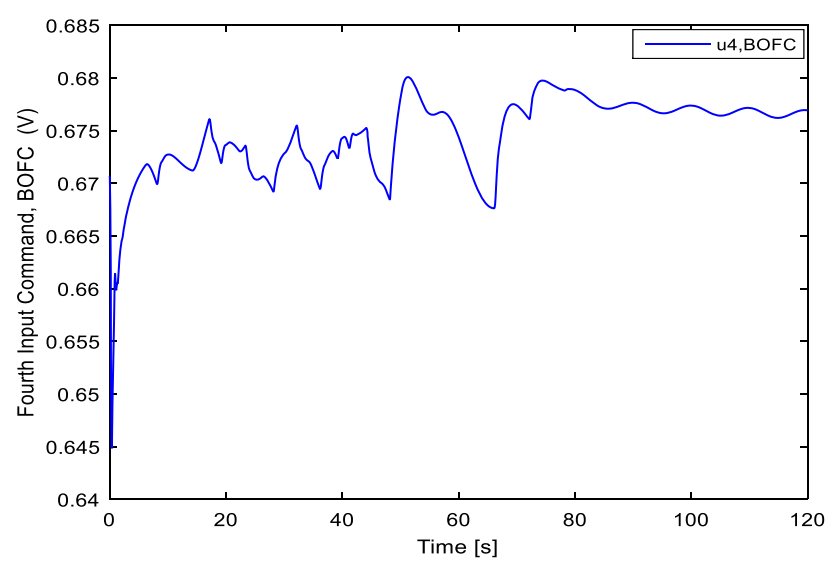

Fig. 21 Fourth input command $u_{4}$ under BOFC accomplished by Backstepping technique

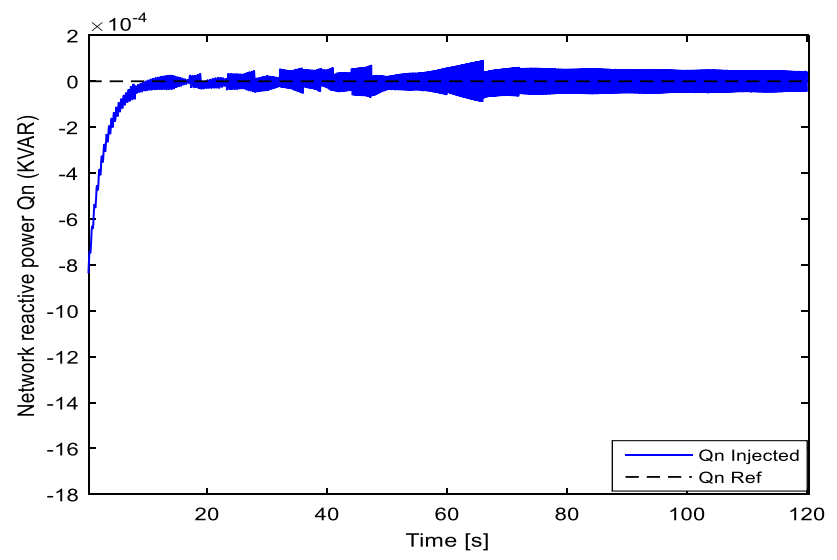

Fig. 22 Dynamic performance of network reactive power regulator (KVAR)

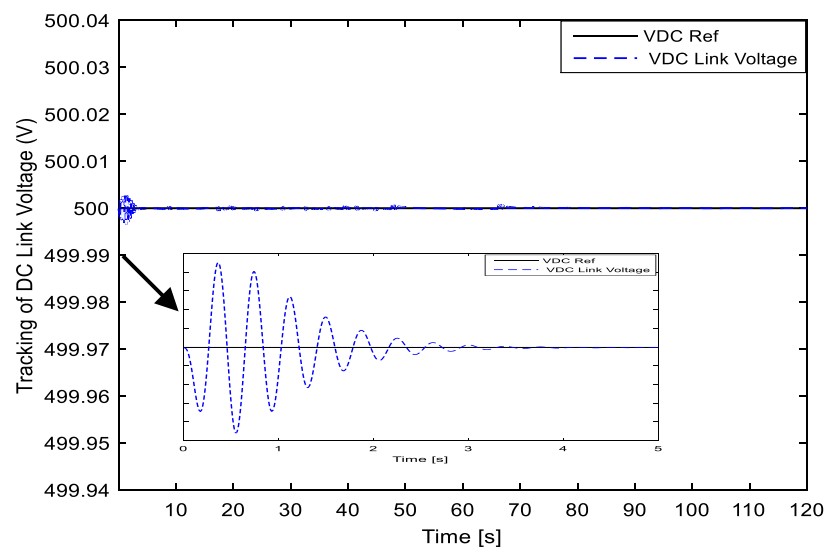

Fig. 23 DC link voltage across the DC capacitor (V)

The DC link voltage is bounded across the DC bus capacitance to make sure that there is no power transfer from the grid side converter through the reverse biasing freewheeling diodes towards generator side converter. Figures 8 and 22 show the development profile of active and reactive powers supplied from DDWTG to the electrical network, respectively.

This study succeeded to increases the maximum admissible sampling period to (60) $\mathrm{ms}$ in case of dynamic high-gain observer design parameter and to improve the performance of high-gain observer with small measurement's noise.

\section{Conclusions}

This paper has been described aspects of the design and implementation of semi-global output feedback control of (7.5) $\mathrm{kW}$ variable speed grid connected drive direct wind turbine generator system to have optimum performance at nominal tip speed ratio and allow the DDWTG system running at best generator rotor speed. In order to design the control algorithm of the actual DDWTG system, it is necessary to reproduce the dynamic performances of PMSG. After the analysis of the aerodynamic, mechanical representation model of wind turbine and the electrical model of PMSG, a MATLAB simulator based on back to back PWM converter is proposed in this paper. Based on Park's transformation, four closed-loop accomplished by backstepping control algorithms making use of the Lyapunov control tools are used to drive four practical control laws for double converters PWM principle.

The main purpose of the modeling and sensorless control of direct drive wind turbine generator system is: (1) identify the mechanical power flow and torque when available sufficient wind velocity; (2) identify the power flow of PMSG connected to electrical network, and (3) avoid the need of mechanical sensors whenever all system state variables are inaccessible for measurements.

The analytical study of sampled HGO design shows that convergence of the state estimates to their real states is theoretically ensured provided that the estimate state variables lie into the neighborhood of the real closed-loop trajectories. The high-gain design approach allows building semi-global convergent nonlinear observers such that the initial observer boundaries can be selected randomly in a compact subset of the whole state-space. In order to validate the theoretical results presented in main theorem, the dynamic performances of the proposed semi-global sampled HGO having dynamic design parameter has been demonstrated throughout a sample study dealing with variable speed grid connected DDWTG system. Therefore, sensorless concept for maximum wind power extraction is achieved successfully throughout running the DDWTG for variable speed control strategy without using mechanical sensors for measuring wind velocity and rotor position such as position encoder. Off course, presence like of these sensors may have negative effects on cost, global system size and reliability of operation. 
The Lyapunov Krasovskii candidate functional LKF approach makes possible the design of semi-global output feedback control considering different specifications dealing with stability and stabilization of sampled control systems with applications concerning with several sciences and practical engineering applications.

On other hand, state-feedback control may cause degradation or deterioration of the control performance, expensive control and unreliable behaviors. Consequently, semi-global output feedback process is very important for the reason that in the practical engineering applications, some of the state variables cannot be measured directly throughout measurement devices such as generator rotor speed and rotor position angle.

The simulation results of the demonstrated sample study have been shown that the proposed sampled HGO has fast transient response, which makes control task easier, good input generator mechanical torque rejection due to the disturbing effect and wind turbulence, accurate power tracking response, robustness of observer performance, smooth motion at low generator speed and efficient operation at high rotor angular speed. The simulation results supported the presented main theory and the improvement with respect to semi-global convergence properties and system state estimates.

Furthermore, the analysis of the control structure is developed depending on the advanced chosen power electronic converters such as buck to buck PWM converter. The speed control scheme for DDWTG system has been presented and simulated, showing that this approach offers an excellent performance to place the PMSG at the desired speed regulation. The active power is generated using DC bus voltage regulator and the reactive power is set as the requirements of the electrical grid, and omitted if a unity power factor correction is the demanded.

Acknowledgements This work is partially supported by the ministry of higher education and scientific research in Iraq to carry out this research with original results.

Open Access This article is distributed under the terms of the Creative Commons Attribution 4.0 International License (http://creativecomm ons.org/licenses/by/4.0/), which permits unrestricted use, distribution, and reproduction in any medium, provided you give appropriate credit to the original author(s) and the source, provide a link to the Creative Commons license, and indicate if changes were made.

\section{References}

1. Executive Office of the President (2013) The president's climate action plan. White house washington. www.whitehouse.gov

2. Jia Y, Wang Q, Yang Z (2007) Experimental study of control strategy for wind generation system. IEEE power electronics specialist conference, PESC, pp 1202-1207
3. Dehghan M, Mohamadian M, Varjani AY (2009) A new variablespeed wind energy conversion system using permanent-magnet synchronous generator and z-source inverter. IEEE Trans Energy Convers 24(3):714-724

4. Errami Y, Ouassaid M, Maaroufi M (2011) Modelling and control strategy of PMSG based variable speed wind energy conversion system. International conference on multimedia computing and systems-ICMCS, Ouarzazate, pp 1-6

5. Babu N, Arulmozhivarman P (2013) Wind energy conversion systems-a technical review. J Eng Sci Technol 8(4):493-507

6. Shim H, Seo JH (2000) Recursive observer design beyond the uniform observability. In: Proceedings of the 39th IEEE conference on decision and control, vol 1, pp 809-814

7. Gauthier J, Kupka I (2001) Deterministic observation theory and applications. Cambridge University Press, Cambridge. ISBN 0521 805937

8. Andrieu V, Parly L, Astolfi A (2009) High-gain observers with updated gain and homogeneous correction terms. Automatica 45(2):422-428

9. Ahrens J, Khalil HK (2009) High-gain observers in the presence of measurement noise: a switched-gain approach. Automatica 45:936-943

10. Boizot N, Busvelle E, Gauthier JP (2010) An adaptive high gain observer for nonlinear systems. Automatica 46(9):1483-1488

11. Beltran B, Ahmed-Ali T, Benbouzid ME (2007) Sliding mode power control of variable speed wind energy conversion systems. IEEE Trans Energy Convers 23(2):943-948

12. Barambones $\mathrm{O}$ (2012) Sliding mode control strategy for wind turbine power maximization. J Energ 5(7):2330-2330

13. Loucif M, Boumedine A, Mechernene A (2014) Maximum power point tracking based on backstepping control of wind turbine. Electromicanica Electromachanica Autom 62(3):103-109

14. Hussein M, Senjyu T, Orabi M, Wahab M, Hamada M (2013) Control of a stand-alone variable speed wind energy supply system. Appl Sci 3(2):437-456

15. Senjyu T, Ochi Y, Kikunaga Y, Tokudome M, Yona A, Muhando B, Urasaki N, Funabashi T (2009) Sensorless maximum power point tracking control for wind generation system with squirrel cage induction generator. Renew Energy 34(4):994-999

16. Rocha R (2011) A sensorless control for a variable speed wind turbine operating at partial load. Renew Energy 36(1):132-141

17. Fu D, Xing Y, Mas Y (2010) MPPT of VSCF wind energy conversion system using xtremum control strategy. World non-gridconnected wind power and energy conference (WNWEC), IEEE conference, pp 1-6

18. Kesraoui M, Korichi N, Belkadi A (2011) Maximum power point tracker of wind energy conversion system. Renew Energy Gener Appl 36(10):2655-2662

19. Khalil H, Laurent $P$ (2014) High-gain observers in nonlinear feedback control. Int J Robust Nonlinear Control 24(6):993-1015

20. Dabroom AM, Khalil HK (2001) Output feedback sampled-data control of nonlinear systems using high-gain observers. IEEE Trans Automat Control 46:1712-1725

21. Liu M, Liu Y, Li F (2015) Semi-global stabilization via outputfeedback for a class of non-triangular nonlinear systems with an unknown coefficient. Hindawi Publishing Corporation Mathematical Problems in Engineering, pp 3-8

22. Burton T, Jenkins N, Sharpe D, Bossanyi E (2011) Wind energy handbook, 2nd edn. Wiley, New York

23. Bianchi D, Battista H, Mantz R (2007) Wind turbine control systems: principles, modelling and gain scheduling design. Springer, London

24. El Magri A, Giri F, Abouloifa A, El Fadili A (2009) Nonlinear control of associations including wind turbine PMSG and AC/DC/AC converter-speed regulation and power factor correction. IEEE European control conference, pp 23-26 
25. Moreno JS, Besancon G, Martinez J (2013) Observer-based maximum power tracking in wind turbines with only generator speed measurements. European control conference, Switzerland, pp 478483

26. El Magri A, Giri F, Abouloifa A, Fatima Z (2009) Nonlinear control of associations including synchronous motors and AC/DC/AC converters: a formal analysis of speed regulation and power factor correction. IEEE, American control conference, pp 3470-3475

27. Boumédiène A, Boucherit M, Boudjema F (2007) A robustness comparative study of several types of speed controllers with the kind based on backstepping for pmsm control fed by a three-level voltage source inverter. Int Rev Electr Eng 2(4):619-633

28. Rashid H (2011) Power electronics handbook: devices, circuits, and applications, 3rd edn. Elsevier Inc, New York. ISBN-10: 0123820367

29. Krstic M, Kanellakopoulos I, Kokotovic PV (1995) Nonlinear and adaptive control design. Wiley, New York

30. Koutroulis E (2006) Design of a maximum power tracking system for wind-energy conversion applications. IEEE Trans Industr Electron 53(2):486-494
31. El Magri A, Giri F, El fadili A (2013) AC electric motors control: advanced design techniques. Wiley, Oxford. ISBN: 978-1-11833152-1

32. Hann C (2014) Contribution to the synthesis of observers for nonlinear systems under communication constrains. Ph.D. thesis, UCBN, France

33. Shim H, Seo JH (2000) Nonlinear output feedback stabilization on a bounded region of attraction. Int J Control 73(5):416-426

34. Shim H, Teel AR (2001) On performance improvement of an output feedback control for non-uniformly observable nonlinear systems. In: Proceeding of the 40'th IEEE conference on decision and control, Orlando, USAP, pp 1354-1359

35. Shim H, Young I, Seo JH (2001) Semi-global observer for multioutput nonlinear systems. Syst Control Lett 42(2001):233-244

36. Fridman E (2014) Introduction to time-delay systems: analysis and control. Birkhauser, Basel. ISBN 9783319093932 